Article

\title{
Identification of Chemical Profiles and Biological Properties of Rhizophora racemosa G. Mey. Extracts Obtained by Different Methods and Solvents
}

\author{
Annalisa Chiavaroli ${ }^{1}$, Koaudio Ibrahime Sinan ${ }^{2}$, Gokhan Zengin ${ }^{2, *}$, \\ Mohamad Fawzi Mahomoodally ${ }^{3,4}$ (D), Nabeelah Bibi Sadeer ${ }^{4} \mathbb{D}$, Ouattara Katinan Etienne ${ }^{5}$, \\ Zoltán Cziáky $^{6}{ }^{(D)}$, József Jekő ${ }^{6}$, Jasmina Glamočlija ${ }^{7}$, Marina Soković ${ }^{7}$, Lucia Recinella ${ }^{1}$, \\ Luigi Brunetti ${ }^{1}$, Sheila Leone ${ }^{1}$, Hassan H. Abdullah ${ }^{8,9}$, Paola Angelini 10,*(D), \\ Giancarlo Angeles Flores ${ }^{10}$, Roberto Venanzoni ${ }^{10}(\mathbb{D})$, Luigi Menghini ${ }^{1}\left(\mathbb{D}\right.$, Giustino Orlando ${ }^{1, *}$ \\ and Claudio Ferrante ${ }^{1}$ (D) \\ 1 Department of Pharmacy, University “G. d'Annunzio" of Chieti-Pescara, 66100 Chieti, Italy; \\ annalisa.chiavaroli@unich.it (A.C.); lucia.recinella@unich.it (L.R.); luigi.brunetti@unich.it (L.B.); \\ sheila.leone@unich.it (S.L.); luigi.menghini@unich.it (L.M.); claudio.ferrante@unich.it (C.F.) \\ 2 Department of Biology, Science Faculty, Selcuk Universtiy, Campus Konya, 42130 Konya, Turkey; \\ sinankouadio@gmail.com \\ 3 Institute of Research and Development, Duy Tan University, Da Nang 550000, Vietnam; \\ mohamadfawzimahomoodally@duytan.edu.vn or f.mahomoodally@uom.ac.mu \\ 4 Department of Health Sciences, Faculty of Science, University of Mauritius, Réduit 230, Mauritius; \\ nabeelah.sadeer1@umail.uom.ac.mu \\ 5 Laboratoire de Botanique, UFR Biosciences, Université Félix Houphouët-Boigny, 01 Abidjan, Ivory Coast; \\ katinan.etienne@gmail.com \\ 6 Agricultural and Molecular Research and Service Institute, University of Nyíregyháza, 4400 Nyíregyháza, \\ Hungary; cziaky.zoltan@nye.hu (Z.C.); jjozsi@gmail.com (J.J.) \\ 7 Department of Plant Physiology, Institute for Biological Research "Siniša Stanković”, University of Belgrade, \\ 11000 Belgrade, Serbia; jasna@ibiss.bg.ac.rs (J.G.) marina.sokovic@mpn.gov.rs (M.S.) \\ 8 Chemistry Department, College of Education, Salahaddin University-Erbil, Erbil 44001, Iraq; \\ hassan.abdullah@su.edu.krd \\ 9 School of Pharmaceutical Sciences, Universiti Sains Malaysia, USM, Penang 11800, Malaysia \\ 10 Department of Chemistry, Biology and Biotechnology, University of Perugia, 06100 Perugia, Italy; \\ giancarlo.angelesflore@studenti.unipg.it (G.A.F.); roberto.venanzoni@unipg.it (R.V.) \\ * Correspondence: gokhanzengin@selcuk.edu.tr (G.Z.); paola.angelini@unipg.it (P.A.); \\ giustino.orlando@unich.it (G.O.)
}

Received: 22 May 2020; Accepted: 16 June 2020; Published: 18 June 2020

\begin{abstract}
Mangrove forests exemplify a multifaceted ecosystem since they do not only play a crucial ecological role but also possess medicinal properties. Methanolic, ethyl acetate and aqueous leaf and bark extracts were prepared using homogenizer-assisted extraction (HAE), infusion and maceration (with and without stirring). The different extracts were screened for phytochemical profiling and antioxidant capacities in terms of radical scavenging (DPPH, ABTS), reducing potential (CUPRAC, FRAP), total antioxidant capacity and chelating power. Additionally, R. racemosa was evaluated for its anti-diabetic ( $\alpha$-amylase, $\alpha$-glucosidase), anti-tyrosinase and anti-cholinesterase (AChE, BChE) activities. Additionally, antimycotic and antibacterial effects were investigated against Eescherichia coli, Pseudomonas aeruginosa, Salmonella typhimurium, Listeria monocytogenes, Enterobacter cloacae, Bacillus cereus, Micrococcus luteus, Staphylococcus aureus, Aspergillus fumigatus, Aspergillus niger, Trichoderma viride, Penicillium funiculosum, Penicillium ochrochloron and Penicillium verrucosum. Finally, based on phytochemical fingerprint, in silico studies, including bioinformatics, network pharmacology and docking approaches were conducted to predict the putative targets, namely tyrosinase, lanosterol-14- $\alpha$-demethylase and E. coli DNA gyrase, underlying the observed
\end{abstract}


bio-pharmacological and microbiological effects. The methanolic leave and bark extracts (prepared by both HAE and maceration) abounded with phenolics, flavonoids, phenolic acids and flavonols. Results displayed that both methanolic leaf and bark extracts (prepared by HAE) exhibited the highest radical scavenging, reducing potential and total antioxidant capacity. Furthermore, our findings showed that the highest enzymatic inhibitory activity recorded was with the tyrosinase enzyme. In this context, bioinformatics analysis predicted putative interactions between tyrosinase and multiple secondary metabolites including apigenin, luteolin, vitexin, isovitexin, procyanidin B, quercetin and methoxy-trihydroxyflavone. The same compounds were also docked against lanosterol-14 $\alpha$-demethylase and E. Coli DNA gyrase, yielding affinities in the submicromolar-micromolar range that further support the observed anti-microbial effects exerted by the extracts. In conclusion, extracts of $R$. racemosa may be considered as novel sources of phytoanti-oxidants and enzyme inhibitors that can be exploited as future first-line pharmacophores.

Keywords: mangrove; phytochemical; homogenizer-assisted extraction; phytoanti-oxidants; enzyme inhibition; anti-microbial activity; in silico studies

\section{Introduction}

Mangrove forests are the most productive and richest ecosystems on the planet. They provide valuable ecological benefits to the coastal lines of tropical and sub-tropical countries by acting as a protective barrier against waves, purify the nearby marine environment and provide habitats for countless reptiles, birds and fish [1]. Besides their perennial ecological roles, mangroves hold another important facet, which we sometimes overlook-the therapeutic value possessed by the plants. The intuitive nature of humans believed that the different parts of mangroves can cure a variety of diseases, namely rheumatism, diabetes, snake bites, asthma, skin diseases, throat pains, diarrhea, fever, intestinal worms, among others [2,3]. However, most of these traditional beliefs have not been verified yet, which subsequently triggers a dire need for a thorough investigation. Indeed, as supported by one of our recent comprehensive review on mangroves, it is mentioned that although a total of 84 mangrove species are recorded to-date, only 27 of them have been validated for their pharmaceutical effects [4].

This considerable meagerness of studies on the pharmacological aspects of mangroves has not changed during the past decades and it is evident by a lack of published work regarding this matter. Therefore, to fill this niche, we focus our work on an untapped mangrove species named Rhizophora racemosa G. Mey. (Family: Rhizophoraceae) by evaluating its pharmacological properties and identifying phytochemicals present. Morphologically, $R$. racemosa is a tree reaching a height of up to $30 \mathrm{~m}$ developing stilt roots and elliptical leaves. This species has the potential to bloom 128 flowers on one axillary branch. Sepals of flowers are $8-10 \mathrm{~mm}$ long [5]. The Nigerian people traditionally used the leaves of $R$. racemosa to treat toothache and dysmenorrhea. A study was conducted to determine the lethal dose (LD50) of the methanolic leaf extract. Results showed that the LD50 of the extract was $1583.33 \mathrm{mg} / \mathrm{kg}$ which is considered safe for consumption [6]. In Benin, the roots of the mangrove plant, locally called Wéto, is used to manage malaria [7].

In a revision of phytochemical knowledge of the genus [8], it is reported that the species R. racemosa from Australia contains primary and secondary metabolites, such as sugars and polysaccharides, aminoacids as well as polyphenols, triterpenes and tannins. The phytochemical profile of water extract from the bark of $R$. racemosa includes saponins and terpenes and possesses anti-microbial activity against the phytopathogen fungus Lasiodiplodia theobromae [9]. The presence of multiple classes of secondary metabolites was qualitatively confirmed [10].

Out of the seven accepted members of the Rhizophora genus [11], R. racemosa is considered as underexplored since as far as our literature could establish, we noticed a dearth of information with no attempt in evaluating the antioxidant properties and enzymatic inhibitory effects of that particular 
mangrove species. Thus, our present study aims at shedding more light on this poorly understood plant. Our work is as follows: (1) characterize the phytochemicals present in leaves and barks using in vitro standard assays, (2) assess antioxidant properties in terms of radical scavenging, reducing potential, total antioxidant capacity and metal chelating, (3) evaluate the enzymatic inhibitory effects related to chronic diseases namely diabetes mellitus type II, neurodegenerative complications and skin disorders and (4) make a comparison between different extraction methods (infusion, homogenizer assisted extraction, maceration (with and without stirring)) used to prepare the samples. Additionally, antimycotic and antibacterial effects were investigated against Escherichia coli, Pseudomonas aeruginosa, Salmonella typhimurium, Listeria monocytogenes, Enterobacter cloacae, Bacillus cereus, Micrococcus luteus, Staphylococcus aureus, Aspergillus fumigatus, Aspergillus niger, Trichoderma viride, Penicillium funiculosum, Penicillium ochrochloron and Penicillium verrucosum. Finally, based on phytochemical fingerprint, in silico studies, including bioinformatics, network pharmacology and docking approaches were conducted to predict the putative targets underlying the observed bio-pharmacological and microbiological effects.

\section{Materials and Methods}

\subsection{Plant Material and Preparation of Extracts}

R. racemosa was collected in Mafiblé village, municipality of Port-Bouët, city of Abidjan of Côte d'Ivoire, in the year 2019 and it was authenticated by the botanist Ouattara Katinan Etienne (Université Félix Houphouet Boigny, Abidjan, Côte d'Ivoire). The plant materials were dried in the shade in an air-ventilated environment (about 10 days). The leaves and stem barks were carefully separated. These plant materials were powdered by using a laboratory mill. The powdered plant materials were stored in the dark at $20^{\circ} \mathrm{C}$.

We used homogenizer-assist extraction (HAE), maceration (with stirred and without stirred) and infusion techniques in the present study. Ethyl acetate and methanol were used for HAE and maceration techniques. Water was used in the infusions. The flowchart is given in Figure 1. Obtained extracts were filtered and then evaporated by using a rotary evaporator. Infusions were lyophilized and all dried extracts were stored at $4{ }^{\circ} \mathrm{C}$ until analysis.

\subsection{Profile of Bioactive Compounds}

The total phenolic, phenolic acid, flavanol and flavonoid contents of the extracts were measured and detailed methods were described in our previous paper [12,13]. Standards, namely gallic acid (GAE) for phenolics, caffeic acid (CE) for phenolic acid, catechin (CAE) for flavanol and rutin (RE) for flavonoids, were used to explain the results.

Gradient reversed-phase ultra high performance liquid chromatography (UHPLC) separations with electrospray MS/MS detection (both positive and negative ion modes) were used for the structural characterization of the compounds presenting in different extracts. The UHPLC system consisted of the Dionex Ultimate (Thermo Scientific, Waltham, MA, USA) 3000RS UHPLC instrument coupled to a Thermo Q Exactive Orbitrap mass spectrometer (Thermo Scientific, Waltham, MA, USA). Chromatographic separation was achieved on a reversed-phase column Thermo Accucore (Thermo Scientific, Waltham, MA, USA) C18 (100 mm $\times 2.1 \mathrm{~mm}$ i. d., $2.6 \mu \mathrm{m})$ [14]. All analytical details were given in Supplementary Material.

\subsection{Determination of Antioxidant and Enzyme Inhibitory Effects}

To detect antioxidant properties, we used several chemical assays including different mechanisms namely, radical scavenging ((2,2'-azino-bis (3-ethylbenzothiazoline-6-sulphonic acid) (ABTS), and 2,2-diphenyl-1-picrylhydrazyl (DPPH)), reducing power (ferric reducing power (FRAP, transformation from $\mathrm{Fe}^{3+}$ to $\mathrm{Fe}^{2+}$ by anti-oxidants), cupric reducing power (CUPRAC, transformation from $\mathrm{Cu}^{2+}$ to $\mathrm{Cu}^{+}$by anti-oxidants)), phosphomolybdenum and metal chelating. Trolox (TE) and ethylenediaminetetraacetic acid (EDTA) were used as standard antioxidant compounds. Obtained 
results were expressed as equivalents of the standard compounds [15]. All experimental details were given in Supplemental Materials.

To detect inhibitory effects on enzymes, we used colorimetric enzyme inhibition assays and these assays included tyrosinase, $\alpha$-glucosidase, $\alpha$-amylase, and cholinesterases (acetylcholinesterase (AChE), butyrylcholinesterase (BChE)). Some standard inhibitors (galantamine for cholinesterases; kojic acid for tyrosinase and acarbose for $\alpha$-glucosidase, $\alpha$-amylase) were used as positive controls. The results were expressed as equivalents of the standard compounds. All experimental details were given in Supplemental Materials.

\subsection{Antibacterial and Anti-fungal Activities}

The Microdilution method was used to evaluate antibacterial and anti-fungal properties of the extracts following methods described in our earlier study [16]. Staphylococcus aureus (ATCC (American Type Culture Collection, Manassas, VA, USA) 6538), Listeria monocytogenes (NCTC 7973), and Bacillus cereus (clinical isolate) were used as Gram-positive bacteria. Salmonella typhimurium (ATCC 13311), Pseudomonas aeruginosa (ATCC 27853), Enterobacter cloacae (human isolate), and Escherichia coli (ATCC 35210) were used as Gram-negative bacteria.

Fungi, namely, Aspergillus fumigatus (human isolate), Aspergillus ochraceus (ATCC 12066), Aspergillus niger (ATCC 6275), Aspergillus versicolor (ATCC 11730), Trichoderma viride (IAM 5061), Penicillium funiculosum (ATCC 36839), Penicillium ochrochloron (ATCC 9112) and Penicillium verrucosum var. cyclopium (food isolate), were used to investigate the anti-fungal properties of the extracts.

Anti-microbial results were evaluated by minimum inhibitory (MIC) and minimum bactericidal/ fungicidal (MBC/MFC) concentrations. Ampicillin and Streptomycin were used as standards for antibacterial activity. Bifonazole and ketoconazole were used as positive controls for anti-fungal evaluation.

\subsection{Bioinformatics and Docking Studies}

Chemical structures were prepared and converted into canonical. The simplified molecular-input line-entry system (SMILES) using ChemSketch software (Advanced Chemistry Development, Inc., (ACD/Labs), Toronto, ON, Canada). The SMILES were then processed by the SwissTargetPrediction (http://www.swisstargetprediction.ch/) platform, for predicting putative protein targets. The names of the identified targets were normalized according to the UniProt database (https://www.uniprot.org/). Cytoscape software (3.7.2 version; National Institute of General Medical Sciences (NIGMS), Bethesda, MD, USA) was used to create a Venn diagram of identified phytochemicals in the tested extracts and a components-targets illustration network.

Regarding the docking analysis, the routine steps for docking calculations involve the preparation of the inhibitors and the protein. The crystal structures of the proteins were downloaded from Protein Data Bank (PDB). The PDB codes were: 5M8P; 6RKS; 4LXJ. To prepare the protein for docking calculations, all water molecules and co-crystallized compounds were removed. This step was followed by adding polar hydrogen atoms and neutralized using the Autodock4 program (Molinspiration Database). The starting structures of secondary metabolites were optimized to their ground state structures using the AM1 semiempirical method and the 3D structures were saved in mol2 format. The protein was immersed in a 3D grid box with $60 \times 60 \times 60$ dimensions with $0.375 \AA$ distance between points. A Lamarckian genetic algorithm was used to calculate the docking free energy of 250 confirmations for each inhibitor. The docking results were clustered and organized according to the docking free energy. The binding site was localized, and the non-bonding interactions were elucidated using Discovery Studio 5.0 visualizer.

\subsection{Statistical Analysis}

Results were done as a mean \pm standard deviation (SD). One-way analysis of the variance was used to evaluate significant differences among samples $(p<0.05$, Turkey's post hoc test) for each 
assay done. Principal component analysis was performed to visualize the (dis)similarity between the samples and k-medoids cluster analysis was used for the classification. The performance of k-medoids clustering was assessed by estimating the silhouette coefficient $\left(S_{i}\right)$. A value of $S_{i}$ close to 1 indicates a good clustered.

The dataset was submitted to Supervised PLS-DA (Sparse Partial Least Squares) in an attempt to discriminate $R$. racemosa organs. Model goodness was recorded by estimating area under curve (AUC) value. Variable importance in projection was achieved to determine the influence of each biological activity in organ separation. Biological activities with variable importance of projection (VIP score) $>1.1$ were considered to have the highest discrimination potential (VIP score $>1$ ). R v 3.6.1 statistical software was used for the analysis (R Core Team, Vienna, Austria).

\section{Results}

\subsection{Profiling of Bioactive Compounds}

Phenolic compounds are crucial for the physiology of both plants and human beings since they provide a protective role in the human body by acting as anti-oxidants against oxidative stress [17]. Furthermore, literature previously reported important biological activities, namely anti-cancer, anti-inflammatory, anti-microbial, anti-diabetic and neuroprotective [18-20]. Therefore, the quantitative estimation of phytochemicals of different extracts of the leaves and barks of $R$. racemosa was undertaken. The results are summarized in Table 1. Three different extraction techniques, namely infusion, maceration and homogenizer-assisted extraction (HAE) were employed to extract potential bioactive compounds. Among the aforementioned techniques, HAE is the most recommended one since it consumes a low amount of solvent and time and is environment friendly [21]. Indeed, our results revealed that the methanolic leaf extracts prepared by HAE yielded higher phenolic, phenolic acids and flavonol contents (217.21 mg GAE/g, $58.50 \mathrm{mg}$ CAE/g and $62.43 \mathrm{mg}$ CE/g, respectively). Likewise, the same technique extracted the highest quantity of phenolic and flavonol contents $(210.00 \mathrm{mg}$ GAE $/ \mathrm{g}$ and $66.17 \mathrm{mg} \mathrm{CE} / \mathrm{g}$, respectively) from the methanolic bark extract. In terms of flavonoid extraction, infusion was the best technique yielding the highest amount (15.01 mg RE/g) from methanolic bark while maceration method extracted the highest amount from methanolic leaf extract (38.45 mg RE/g). Irrespective of the extraction methods used, leaf possessed the highest amount of phenolic and flavonoid in contrast to bark. The bark yielded the highest amount of phenolic acid and flavonol compared to leaf (Table 1).

The identification of compounds was based on their retention time, the accurate mass and the registered mass spectra fragmentation patterns. The UHPLC method used was very versatile and allows for the separation of components in the medium to low polarity range, however, it had limited resolving power for polar compounds (e.g., sugars, short-chain amino acids and organic acids), which generally elute in the void volume. A detailed list for chemical profiles is given in Supplemental Materials.

Phenolic acids, flavonoids, di-, tri- tetra- and pentahydroxyflavone and flavanone derivatives, procyanidins and catechins were mostly identified in the extracts. Finally, 38-60 compounds were characterized in the extracts by comparing our LC-MS/MS data with data from the literature and with our previous results. We could identify a wide range of compounds e.g., lower molecular weight polar component (hydroxybenzaldehyde, molecular weight (MW): 122) or a catechin trimer (MW: 866) or flavon and flavanone derivatives (MW: 4-600). The most 56/60 compounds were detected, as expected, in methanol extracts, while the least compound was detected in ethyl acetate extracts. The leaves and bark were found to have a similar qualitative composition independently from extraction methods.

Many quinic acid derivatives were observed in the extracts. Components with the retention time 10.24, and 14.88 were assigned as chlorogenic acid and chlorogenic acid isomer according to their $[\mathrm{M}+\mathrm{H}]^{+}$ions at $\mathrm{m} / \mathrm{z} 355.1029$ and they showed characteristic product ions. Chlorogenic acid was identified by comparison with an authentic standard. These compounds were detectable in 
both polarities. 4-Coumaric acid was identified in the extracts in negative ion mode (18.54 $\mathrm{min}, \mathrm{m} / \mathrm{z}$ 163.03952), by comparisons with a reference standard (Table 2).

Flavanol monomers catechin/epicatechin (retention time 14.00/17.61 min) were identified by molecular ion $[\mathrm{M}-\mathrm{H}]^{-} \mathrm{m} / \mathrm{z} 289.07121$ and their fragment ions. Catechin dimer, procyanidin $\mathrm{B}$, was detected at $15.69 \mathrm{~min}$ by molecular ion $[\mathrm{M}-\mathrm{H}]^{-} \mathrm{m} / \mathrm{z} 577.1346$ and catechin trimer, procyanidin $\mathrm{C}$, was detected at $17.33 \mathrm{~min}$ by molecular ion $[\mathrm{M}-\mathrm{H}]^{-} \mathrm{m} / \mathrm{z} 865.19799$.

Most of the flavonoids were detected as glycoconjugates, such as hexose, pentose and dihexose. Characteristic mass losses of $C$ - and $O$-glycosides were observed in their MS/MS spectra.

Compounds with the retention time 19.30, 21.70, 22.71 and $25.44 \min \left(\mathrm{C}_{24} \mathrm{H}_{20} \mathrm{O}_{9}\right)$, displaying the same MS fragmentation, were identified as cinchonain I isomers. Compounds with retention time $16.08,17.04,17.91$ and $18.09 \min \left(\mathrm{C}_{39} \mathrm{H}_{32} \mathrm{O}_{15}\right)$ shared the same MS fragmentation pattern, correspond to cinchonain II type compounds. We could detect cinchonain type compounds in all extracts by their deprotonated molecular ions, but we confirmed the presence of compounds and listed in the Tables if we could record at least five characteristic fragments.

\subsection{Antioxidant Activities}

The methanolic, ethyl acetate and aqueous extracts of the two plant parts (leaves and barks) were subjected to six different antioxidant assays. We screened our different extracts using multiple assays in terms of their radical scavenging ability (DPPH and ABTS), reducing potential (CUPRAC and FRAP), total antioxidant capacity and metal chelating power. The collected data are summarized in Table 3 .

Herein, our findings demonstrated that the leaves extracts prepared from HAE (ethyl acetate and methanolic), maceration (ethyl acetate and methanolic) and maceration (with and without stirring) exhibited relatively the same $\mathrm{AChE}$ activities with galantamine equivalent ranging from 8.35 to $8.78 \mathrm{mg} / \mathrm{g}$. Likewise, the same trend was observed with bark extract resulting in galantamine equivalent ranging from 8.04 to $8.76 \mathrm{mg} / \mathrm{g}$. However, extracts (both leaf and bark) prepared from infusion projected the least AChE inhibitory effect (Table 4). In terms of BChE inhibition, a different trend was observed whereby several inactivities were even reported with a few extracts irrespective of the extraction technique used. The ethyl acetate leaf extract (maceration without stirring) exhibited the highest BChE activity (3.50 mg GALAE/g) while aqueous leaf extract displayed the lowest activity (0.91 mg GALAE/g). On the other hand, ethyl acetate bark extract (maceration with stirring) showed the highest BChE inhibitory effect (3.03 mg GALAE/g).

Blemished skin, acne scars, age spots, melasma and post-inflammatory hyperpigmentation do not only occur during puberty but equally affect adults. These skin problems are caused by an increase in melanin production and the enzyme responsible is tyrosinase. Therefore, searching for tyrosinase inhibitors are highly recommended to fight against these skin disorders [23]. Herein, we screened our extracts for tyrosinase inhibitory effects. Results showed that methanolic leaf and bark both prepared by maceration displayed the highest tyrosinase inhibition (146.76 and $154.33 \mathrm{mg} \mathrm{KAE} / \mathrm{g}$, respectively). While the lowest activity was recorded with bark infusion (25.64 $\mathrm{mg} \mathrm{KAE} / \mathrm{g}$ ), no activity was noted with leaf infusion (Table 4).

It is important to highlight that the methanolic leaf and bark extracts prepared from the extraction technique, HAE, revealed the highest antioxidant properties with DPPH, ABTS, CUPRAC, FRAP and phosphomolybdenum assays. On the other hand, ethyl acetate leaves and bark extracts prepared from maceration (without stir) possessed the most potent chelating power. For instance, with the DPPH assay which involved the reduction of a ferric-tripyridyl triazine complex to its colored ferrous form in the presence of anti-oxidants, the methanolic leaf and bark extracts showed highest DPPH radical scavenging ability (525.84 and $512.37 \mathrm{mg}$ TE/g, respectively). A similar trend was observed with ABTS assay classifying both the methanolic leaf and bark extracts as the most potent ABTS radical scavengers (600.84 and $581.39 \mathrm{mg} \mathrm{TE} / \mathrm{g}$, respectively). 
Table 1. Total bioactive compounds (phenolic (TPC), flavonoid (TFC), phenolic acid (TPAC) and flavonol (TFlv) of the tested extracts *.

\begin{tabular}{|c|c|c|c|c|c|}
\hline Parts & Methods-Solvents & TPC (mg GAE/g) & TFC (mg RE/g) & TPAC (mg CAE/g) & TFlv (mg CE/g) \\
\hline \multirow{7}{*}{ Leaves } & infusion & $83.85 \pm 1.12^{f}$ & $17.22 \pm 0.30^{\mathrm{e}}$ & $26.40 \pm 0.77^{\mathrm{d}}$ & $1.66 \pm 0.01 \mathrm{ij}$ \\
\hline & HAE-EA & $50.17 \pm 0.57 \mathrm{~g}$ & $24.06 \pm 2.09 \mathrm{~cd}$ & nd & $8.28 \pm 0.03^{f}$ \\
\hline & HAE-MEOH & $217.21 \pm 0.69^{\mathrm{a}}$ & $33.10 \pm 1.78^{b}$ & $58.50 \pm 2.03^{a}$ & $62.43 \pm 0.41^{b}$ \\
\hline & Maceration EA & $35.44 \pm 0.32^{h}$ & $25.57 \pm 0.75^{c}$ & nd & $2.92 \pm 0.06 \mathrm{hi}$ \\
\hline & Maceration-MEOH & $198.57 \pm 5.51^{\mathrm{c}}$ & $38.45 \pm 0.75^{\mathrm{a}}$ & $49.69 \pm 2.35^{b}$ & $60.42 \pm 0.36^{c}$ \\
\hline & Maceration (not stirred)-EA & $24.27 \pm 0.40^{j}$ & $22.20 \pm 0.35^{\mathrm{d}}$ & nd & $1.46 \pm 0.02 \mathrm{ij}$ \\
\hline & Maceration (not stirred)-MEOH & $191.74 \pm 0.64^{d}$ & $38.07 \pm 0.60^{\mathrm{a}}$ & $40.39 \pm 7.11^{\mathrm{c}}$ & $58.11 \pm 0.98^{\mathrm{d}}$ \\
\hline \multirow{7}{*}{ Bark } & infusion & $145.15 \pm 0.39 \mathrm{e}$ & $15.01 \pm 1.76^{\text {ef }}$ & $61.50 \pm 1.65^{\mathrm{a}}$ & $5.27 \pm 0.08 \mathrm{~g}$ \\
\hline & HAE-EA & $30.34 \pm 0.33^{\mathrm{i}}$ & $7.96 \pm 0.10^{\mathrm{i}}$ & nd & $3.81 \pm 0.02 \mathrm{gh}$ \\
\hline & HAE-MEOH & $210.00 \pm 1.28^{b}$ & $9.00 \pm 0.52 \mathrm{hi}$ & $47.73 \pm 0.93^{b}$ & $66.17 \pm 1.40^{\mathrm{a}}$ \\
\hline & Maceration EA & $30.84 \pm 0.18 \mathrm{hi}$ & $8.81 \pm 0.20 \mathrm{hi}$ & nd & $2.29 \pm 0.06^{\text {hij }}$ \\
\hline & Maceration-MEOH & $201.73 \pm 0.43^{c}$ & $13.64 \pm 0.79 \mathrm{fg}$ & $34.04 \pm 0.41^{\mathrm{c}}$ & $63.21 \pm 1.20^{b}$ \\
\hline & Maceration (not stirred)-EA & $28.51 \pm 0.26^{\mathrm{ij}}$ & $10.96 \pm 0.45 \mathrm{gh}$ & nd & $0.73 \pm 0.01 \mathrm{j}$ \\
\hline & Maceration (not stirred)-MEOH & $192.92 \pm 0.74^{d}$ & $12.52 \pm 0.15 \mathrm{fg}$ & $26.70 \pm 2.74 \mathrm{~d}$ & $11.59 \pm 0.09 \mathrm{e}$ \\
\hline
\end{tabular}

assisted extraction; EA: Ethyl acetate; MEOH: Methanol. Different letters (a-j) indicate significant differences in the extracts $(p<0.05)$. 
Table 2. Chemical composition of the tested extracts.

\begin{tabular}{|c|c|c|c|c|c|c|c|c|}
\hline No. & Name & Formula & $\begin{array}{l}\text { Leaves } \\
\text { Infusion }\end{array}$ & $\begin{array}{c}\text { Leaves } \\
\text { HAE-EA }\end{array}$ & $\begin{array}{c}\text { Leaves } \\
\text { HAE-MeOH }\end{array}$ & $\begin{array}{c}\text { Bark } \\
\text { Infusion }\end{array}$ & $\begin{array}{c}\text { Bark } \\
\text { HAE-EA }\end{array}$ & $\begin{array}{c}\text { Bark } \\
\text { HAE-MeOH }\end{array}$ \\
\hline 1 & Gallic acid (3,4,5-Trihydroxybenzoic acid) & $\mathrm{C}_{7} \mathrm{H}_{6} \mathrm{O}_{5}$ & + & - & - & + & - & + \\
\hline 2 & Protocatechuic acid (3,4-Dihydroxybenzoic acid) & $\mathrm{C}_{7} \mathrm{H}_{6} \mathrm{O}_{4}$ & + & + & + & + & + & + \\
\hline 3 & Pantothenic acid & $\mathrm{C}_{9} \mathrm{H}_{17} \mathrm{NO}_{5}$ & - & - & - & + & - & + \\
\hline 4 & Neochlorogenic acid (5-O-Caffeoylquinic acid) & $\mathrm{C}_{16} \mathrm{H} 18 \mathrm{O} 9$ & + & + & + & + & + & + \\
\hline 5 & Prodelphinidin C & $\mathrm{C}_{30} \mathrm{H}_{26} \mathrm{O}_{13}$ & - & - & - & - & - & + \\
\hline 6 & Hydroxybenzaldehyde & $\mathrm{C}_{7} \mathrm{H}_{6} \mathrm{O}_{2}$ & + & + & + & - & - & - \\
\hline 7 & 3-O-(4-Coumaroyl)quinic acid & $\mathrm{C}_{16} \mathrm{H}_{18} \mathrm{O}_{8}$ & + & + & + & + & + & + \\
\hline $8^{1}$ & Catechin & $\mathrm{C}_{15} \mathrm{H}_{1} 4 \mathrm{O}_{6}$ & + & - & + & + & + & + \\
\hline 9 & Esculetin (6,7-Dihydroxycoumarin) & $\mathrm{C}_{9} \mathrm{H}_{6} \mathrm{O}_{4}$ & + & + & + & + & + & + \\
\hline $10^{1}$ & Chlorogenic acid (3-O-Caffeoylquinic acid) & $\mathrm{C}_{16} \mathrm{H}_{18} \mathrm{O}_{9}$ & + & + & + & + & + & + \\
\hline 11 & 3-O-Feruloylquinic acid & $\mathrm{C}_{17} \mathrm{H}_{20} \mathrm{O}_{9}$ & + & - & + & + & - & + \\
\hline 12 & Caffeic acid & $\mathrm{C}_{9} \mathrm{H}_{8} \mathrm{O}_{4}$ & + & + & + & + & + & + \\
\hline 13 & Procyanidin B & $\mathrm{C}_{30} \mathrm{H}_{26} \mathrm{O}_{12}$ & + & + & + & + & + & + \\
\hline 14 & Cinchonain II isomer 1 & $\mathrm{C}_{39} \mathrm{H}_{32} \mathrm{O}_{15}$ & + & - & + & + & - & + \\
\hline 15 & Chryptochlorogenic acid (4-O-Caffeoylquinic acid) & $\mathrm{C}_{16} \mathrm{H}_{18} \mathrm{O}_{9}$ & + & + & + & + & + & + \\
\hline 16 & Cinchonain II isomer 2 & $\mathrm{C}_{39} \mathrm{H}_{32} \mathrm{O}_{15}$ & + & - & + & + & - & + \\
\hline 17 & 2-Oxindole & $\mathrm{C}_{8} \mathrm{H}_{7} \mathrm{NO}$ & + & + & + & + & + & + \\
\hline 18 & Procyanidin C & $\mathrm{C}_{45} \mathrm{H}_{38} \mathrm{O}_{18}$ & + & + & + & + & + & + \\
\hline 19 & 5-O-(4-Coumaroyl)quinic acid & $\mathrm{C}_{16} \mathrm{H}_{18} \mathrm{O}_{8}$ & + & + & + & + & - & + \\
\hline $20^{1}$ & Epicatechin & $\mathrm{C}_{15} \mathrm{H}_{14} \mathrm{O}_{6}$ & + & + & + & + & + & + \\
\hline 21 & Cinchonain II isomer 3 & $\mathrm{C}_{39} \mathrm{H}_{32} \mathrm{O}_{15}$ & + & - & + & - & - & - \\
\hline 22 & 4-O-(4-Coumaroyl)quinic acid & $\mathrm{C}_{16} \mathrm{H}_{18} \mathrm{O}_{8}$ & + & - & + & + & - & + \\
\hline 23 & Cinchonain II isomer 4 & $\mathrm{C}_{39} \mathrm{H}_{32} \mathrm{O}_{15}$ & - & - & + & - & - & - \\
\hline 24 & Antiarol (3,4,5-Trimethoxyphenol) & $\mathrm{C}_{9} \mathrm{H}_{12} \mathrm{O}_{4}$ & + & + & + & + & + & + \\
\hline 25 & 5-O-Feruloylquinic acid & $\mathrm{C}_{17} \mathrm{H}_{20} \mathrm{O}_{9}$ & + & - & + & + & - & - \\
\hline $26^{1}$ & 4-Coumaric acid & $\mathrm{C}_{9} \mathrm{H}_{8} \mathrm{O}_{3}$ & + & + & + & + & + & + \\
\hline 27 & Tuberonic acid or 12-Hydroxyjasmonic acid & $\mathrm{C}_{12} \mathrm{H}_{18} \mathrm{O}_{4}$ & + & + & + & + & + & + \\
\hline 28 & 4-O-Feruloylquinic acid & $\mathrm{C}_{17} \mathrm{H}_{20} \mathrm{O}_{9}$ & + & - & + & + & - & + \\
\hline 29 & Riboflavin & $\mathrm{C}_{17} \mathrm{H}_{20} \mathrm{~N}_{4} \mathrm{O}_{6}$ & + & - & + & + & - & + \\
\hline 30 & Cinchonain I isomer 1 & $\mathrm{C}_{24} \mathrm{H}_{20} \mathrm{O}_{9}$ & - & - & + & - & + & + \\
\hline 31 & Naringenin-C-hexoside isomer 1 & $\mathrm{C}_{21} \mathrm{H}_{22} \mathrm{O}_{10}$ & + & - & + & + & - & + \\
\hline $32^{1}$ & Taxifolin (Dihydroquercetin) & $\mathrm{C}_{15} \mathrm{H}_{12} \mathrm{O}_{7}$ & + & + & + & + & + & + \\
\hline 33 & Quercetin-O-rhamnosyldihexoside & $\mathrm{C}_{33} \mathrm{H}_{40} \mathrm{O}_{21}$ & + & + & + & + & + & + \\
\hline 34 & Naringenin-C-hexoside isomer 2 & $\mathrm{C}_{21} \mathrm{H}_{22} \mathrm{O}_{10}$ & + & - & + & + & - & + \\
\hline 35 & Quercetin-O-hexosylhexoside & $\mathrm{C}_{27} \mathrm{H}_{30} \mathrm{O}_{17}$ & + & + & + & + & + & + \\
\hline 36 & Naringenin-C-hexoside isomer 3 & $\mathrm{C}_{21} \mathrm{H}_{22} \mathrm{O}_{10}$ & + & - & + & + & - & + \\
\hline
\end{tabular}


Table 2. Cont

\begin{tabular}{|c|c|c|c|c|c|c|c|c|}
\hline No. & Name & Formula & $\begin{array}{c}\text { Leaves } \\
\text { Infusion }\end{array}$ & $\begin{array}{c}\text { Leaves } \\
\text { HAE-EA }\end{array}$ & $\begin{array}{c}\text { Leaves } \\
\text { HAE-MeOH }\end{array}$ & $\begin{array}{c}\text { Bark } \\
\text { Infusion }\end{array}$ & $\begin{array}{c}\text { Bark } \\
\text { HAE-EA }\end{array}$ & $\begin{array}{c}\text { Bark } \\
\text { HAE-MeOH }\end{array}$ \\
\hline 37 & Quercetin-O-dirhamnosylhexoside & $\mathrm{C}_{33} \mathrm{H}_{40} \mathrm{O}_{20}$ & + & + & + & + & - & + \\
\hline 38 & Hexahydroxy(iso)flavone-O-hexoside & $\mathrm{C}_{21} \mathrm{H}_{20} \mathrm{O}_{13}$ & + & - & + & + & - & + \\
\hline 39 & Hexahydroxy(iso)flavone-O-rhamnosylhexoside & $\mathrm{C}_{27} \mathrm{H}_{30} \mathrm{O}_{17}$ & + & + & + & + & + & + \\
\hline 40 & Cinchonain I isomer 2 & $\mathrm{C}_{24} \mathrm{H}_{20} \mathrm{O}_{9}$ & - & - & + & - & + & + \\
\hline 41 & Cinchonain I isomer 3 & $\mathrm{C}_{24} \mathrm{H}_{20} \mathrm{O}_{9}$ & - & - & + & - & + & + \\
\hline $42^{1}$ & Vitexin (Apigenin-8-C-glucoside) & $\mathrm{C}_{21} \mathrm{H}_{20} \mathrm{O}_{10}$ & + & + & + & + & + & + \\
\hline 43 & Kaempferol-O-dirhamnosylhexoside & $\mathrm{C}_{33} \mathrm{H}_{40} \mathrm{O}_{19}$ & + & + & + & - & - & - \\
\hline 44 & Isovitexin (Apigenin-6-C-glucoside) & $\mathrm{C}_{21} \mathrm{H}_{20} \mathrm{O}_{10}$ & + & + & + & + & + & + \\
\hline $45^{1}$ & Naringin (Naringenin-7-O-neohesperidoside) & $\mathrm{C}_{27} \mathrm{H}_{32} \mathrm{O}_{14}$ & + & - & + & + & + & + \\
\hline $46^{1}$ & Isoquercitrin (Quercetin-3-O-glucoside) & $\mathrm{C}_{21} \mathrm{H}_{20} \mathrm{O}_{12}$ & - & - & + & + & + & + \\
\hline $47^{1}$ & Rutin (Quercetin-3-O-rutinoside) & $\mathrm{C}_{27} \mathrm{H}_{30} \mathrm{O}_{16}$ & + & + & + & + & + & + \\
\hline 48 & Quercetin-O-pentoside & $\mathrm{C}_{20} \mathrm{H}_{18} \mathrm{O}_{11}$ & + & + & + & - & - & - \\
\hline 49 & Astragalin (Kaempferol-3-O-glucoside) & $\mathrm{C}_{21} \mathrm{H}_{20} \mathrm{O}_{11}$ & + & + & + & - & - & - \\
\hline 50 & Kaempferol-3-O-rutinoside (Nicotiflorin) & $\mathrm{C}_{27} \mathrm{H}_{30} \mathrm{O}_{15}$ & + & + & + & + & + & + \\
\hline 51 & Cinchonain I isomer 4 & $\mathrm{C}_{24} \mathrm{H}_{20} \mathrm{O}_{9}$ & - & - & + & - & + & + \\
\hline $52^{1}$ & Eriodictyol ( $3^{\prime}, 4^{\prime}, 5,7$-Tetrahydroxyflavanone) & $\mathrm{C}_{15} \mathrm{H}_{12} \mathrm{O}_{6}$ & + & + & + & + & + & + \\
\hline $53^{1}$ & Isorhamnetin-3-O-glucoside & $\mathrm{C}_{22} \mathrm{H}_{22} \mathrm{O}_{12}$ & - & - & + & + & - & - \\
\hline 54 & Tetrahydroxy(iso)flavone-O-rhamnosylhexoside & $\mathrm{C}_{28} \mathrm{H}_{32} \mathrm{O}_{16}$ & + & + & + & + & + & + \\
\hline 55 & Abscisic acid & $\mathrm{C}_{15} \mathrm{H}_{20} \mathrm{O}_{4}$ & + & + & + & + & + & + \\
\hline $56^{1}$ & Quercetin (3,3', 4',5,7-Pentahydroxyflavone) & $\mathrm{C}_{15} \mathrm{H}_{10} \mathrm{O}_{7}$ & + & + & + & + & + & + \\
\hline $57^{1}$ & Naringenin (4',5,7-Trihydroxyflavanone) & $\mathrm{C}_{15} \mathrm{H}_{12} \mathrm{O}_{5}$ & + & + & + & + & + & + \\
\hline $58^{1}$ & Luteolin (3', 4',5,7-Tetrahydroxyflavone) & $\mathrm{C}_{15} \mathrm{H}_{10} \mathrm{O}_{6}$ & + & + & + & + & + & + \\
\hline $59^{1}$ & Kaempferol (3,4',5,7-Tetrahydroxyflavone) & $\mathrm{C}_{15} \mathrm{H}_{10} \mathrm{O}_{6}$ & + & + & + & - & - & - \\
\hline $60^{1}$ & Apigenin $\left(4^{\prime}, 5,7\right.$-Trihydroxyflavone $)$ & $\mathrm{C}_{15} \mathrm{H}_{10} \mathrm{O}_{5}$ & - & + & + & + & + & + \\
\hline $61^{1}$ & Isorhamnetin (3'-Methoxy-3,4',5,7-tetrahydroxyflavone) & $\mathrm{C}_{16} \mathrm{H}_{12} \mathrm{O}_{7}$ & - & + & + & - & + & - \\
\hline 62 & Methoxy-trihydroxy(iso)flavone & $\mathrm{C}_{16} \mathrm{H}_{12} \mathrm{O}_{6}$ & - & + & + & - & + & + \\
\hline 63 & Pinocembrin (5,7-Dihydroxyflavanone) & $\mathrm{C}_{15} \mathrm{H}_{12} \mathrm{O}_{4}$ & - & + & + & - & - & - \\
\hline 64 & Tetrahydroxyxanthone & $\mathrm{C}_{13} \mathrm{H}_{8} \mathrm{O}_{6}$ & - & - & - & + & - & - \\
\hline 65 & Tetrahydroxyxanthone isomer 1 & $\mathrm{C}_{13} \mathrm{H}_{8} \mathrm{O}_{6}$ & - & - & - & - & - & + \\
\hline 66 & Tetrahydroxyxanthone isomer 2 & $\mathrm{C}_{13} \mathrm{H}_{8} \mathrm{O}_{6}$ & - & - & - & - & - & + \\
\hline 67 & Luteolin-7-O-glucoside (Cynaroside) & $\mathrm{C}_{21} \mathrm{H}_{20} \mathrm{O}_{11}$ & - & - & - & + & - & + \\
\hline 68 & Luteolin-7-O-rutinoside (Scolymoside) & $\mathrm{C}_{27} \mathrm{H}_{30} \mathrm{O}_{15}$ & - & - & - & + & - & + \\
\hline 69 & Methoxy-tetrahydroxy(iso)flavone & $\mathrm{C}_{15} \mathrm{H}_{10} \mathrm{O}_{5}$ & - & - & - & + & - & - \\
\hline 70 & Methoxy-trihydroxy(iso)flavone isomer 1 & $\mathrm{C}_{16} \mathrm{H}_{12} \mathrm{O}_{6}$ & - & - & - & + & - & - \\
\hline 71 & Methoxy-trihydroxy(iso)flavone isomer 2 & $\mathrm{C}_{16} \mathrm{H}_{12} \mathrm{O}_{6}$ & - & - & - & + & - & - \\
\hline
\end{tabular}

+: present; - absent. ${ }^{1}$ Confirmed by standard 
Table 3. Antioxidant properties of the tested extracts *.

\begin{tabular}{|c|c|c|c|c|c|c|c|}
\hline Parts & Methods-Solvents & DPPH (mg TE/g) & ABTS (mg TE/g) & $\begin{array}{l}\text { CUPRAC } \\
(\mathrm{mg} \text { TE/g) }\end{array}$ & FRAP (mg TE/g) & $\begin{array}{l}\text { Metal chelating } \\
\text { (mg EDTAE/g) }\end{array}$ & $\begin{array}{l}\text { Phosphomolybdenum } \\
\text { (mmol TE/g) }\end{array}$ \\
\hline \multirow{7}{*}{ Leaves } & infusion & $101.26 \pm 0.24 \mathrm{fg}$ & $144.25 \pm 3.10^{\mathrm{e}}$ & $315.43 \pm 5.58^{g}$ & $200.02 \pm 4.73^{f}$ & $8.08 \pm 0.76^{\mathrm{e}}$ & $2.15 \pm 0.11^{d}$ \\
\hline & HAE-EA & $67.69 \pm 5.49^{h}$ & $89.09 \pm 1.99^{f}$ & $193.20 \pm 3.83^{h}$ & $77.29 \pm 1.16^{\mathrm{g}}$ & $1.95 \pm 0.49^{\mathrm{h}}$ & $2.12 \pm 0.29 \mathrm{~d}$ \\
\hline & HAE-MEOH & $525.84 \pm 1.37^{\mathrm{a}}$ & $600.84 \pm 13.60^{a}$ & $1047.10 \pm 9.45^{b}$ & $544.76 \pm 11.89^{b}$ & $4.50 \pm 0.53 \mathrm{fg}$ & $4.82 \pm 0.42^{a}$ \\
\hline & Maceration EA & $20.97 \pm 0.28^{\mathrm{ij}}$ & $32.41 \pm 6.38 \mathrm{~g}$ & $124.15 \pm 2.58^{\mathrm{i}}$ & $50.25 \pm 1.91 \mathrm{hi}$ & $2.32 \pm 0.50 \mathrm{gh}$ & $2.17 \pm 0.08^{\mathrm{d}}$ \\
\hline & Maceration-MEOH & $105.56 \pm 0.06^{\mathrm{f}}$ & $159.42 \pm 0.12 \mathrm{e}$ & $753.22 \pm 4.81^{\mathrm{f}}$ & $401.16 \pm 5.03^{\mathrm{e}}$ & $4.31 \pm 0.95 \mathrm{fg}$ & $4.29 \pm 0.20^{a b c}$ \\
\hline & Maceration (not stirred)-EA & $39.06 \pm 2.14^{\mathrm{i}}$ & $12.31 \pm 0.29 \mathrm{hi}$ & $84.92 \pm 0.74^{j}$ & $40.54 \pm 0.63 \mathrm{hi}$ & $29.39 \pm 1.03^{a}$ & $2.40 \pm 0.13^{d}$ \\
\hline & Maceration (not stirred)-MEOH & $496.94 \pm 3.30 \mathrm{bc}$ & $519.71 \pm 6.78^{c}$ & $825.71 \pm 25.99$ e & $519.69 \pm 2.96^{c}$ & $3.64 \pm 0.31 \mathrm{fgh}$ & $3.95 \pm 0.44 \mathrm{bc}$ \\
\hline \multirow{6}{*}{ Bark } & infusion & $318.53 \pm 8.82 \mathrm{e}$ & $353.91 \pm 11.81^{\mathrm{d}}$ & $766.83 \pm 7.23^{f}$ & $398.10 \pm 6.46^{\mathrm{e}}$ & $18.06 \pm 1.29^{c}$ & $3.63 \pm 0.14^{c}$ \\
\hline & HAE-MEOH & $512.37 \pm 7.15^{\mathrm{ab}}$ & $581.39 \pm 6.42^{\mathrm{a}}$ & $1129.33 \pm 8.62^{a}$ & $633.53 \pm 8.97^{a}$ & $5.42 \pm 0.06^{\mathrm{f}}$ & $4.74 \pm 0.17 \mathrm{ab}$ \\
\hline & Maceration EA & $15.86 \pm 1.83^{\mathrm{ij}}$ & $29.36 \pm 1.37 \mathrm{gh}$ & $126.72 \pm 4.57^{\mathrm{j}}$ & $55.45 \pm 0.32^{h}$ & $10.88 \pm 1.41^{\mathrm{d}}$ & $1.68 \pm 0.15^{\mathrm{d}}$ \\
\hline & Maceration-MEOH & $478.39 \pm 21.72^{c}$ & $555.13 \pm 8.47^{b}$ & $931.13 \pm 18.04^{d}$ & $559.27 \pm 2.63^{b}$ & $5.61 \pm 0.16^{\mathrm{f}}$ & $4.51 \pm 0.36^{\mathrm{ab}}$ \\
\hline & Maceration (not stirred)-EA & na & $7.26 \pm 0.10^{\mathrm{i}}$ & $74.82 \pm 0.34 j$ & $37.67 \pm 1.60^{\mathrm{i}}$ & $24.93 \pm 0.24 b$ & $2.13 \pm 0.17^{\mathrm{d}}$ \\
\hline & Maceration (not stirred)-MEOH & $450.51 \pm 15.42^{d}$ & $513.29 \pm 8.26^{c}$ & $961.26 \pm 1.49^{c}$ & $422.30 \pm 12.50^{\mathrm{d}}$ & $4.89 \pm 0.18^{\mathrm{f}}$ & $4.25 \pm 0.53^{\mathrm{abc}}$ \\
\hline
\end{tabular}

* Values are reported as mean \pm S.D. TE: Trolox equivalent; EDTAE: EDTA equivalent; na: not active. HAE: Homogenizer assisted extraction; EA: Ethyl acetate; MEOH: Methanol. Different

letters $(a-j)$ indicate significant differences in the extracts $(p<0.05)$. 
Table 4. Enzyme inhibitory effects of the tested extracts *

\begin{tabular}{|c|c|c|c|c|c|c|}
\hline Parts & Methods-Solvents & $\begin{array}{l}\text { AChE Inhibition } \\
\text { (mg GALAE/g) }\end{array}$ & $\begin{array}{l}\text { BChE Inhibition } \\
\text { (mg GALAE/g) }\end{array}$ & $\begin{array}{l}\text { Tyrosinase } \\
\text { Inhibition } \\
\text { (mg KAE/g) }\end{array}$ & $\begin{array}{c}\text { Amylase } \\
\text { Inhibition } \\
\text { (mmol ACAE/g) }\end{array}$ & $\begin{array}{c}\text { Glucosidase } \\
\text { Inhibition } \\
\text { (mmol ACAE/g) }\end{array}$ \\
\hline \multirow{7}{*}{ Leaves } & infusion & $5.18 \pm 0.08^{d}$ & $0.91 \pm 0.07^{\mathrm{d}}$ & na & $0.15 \pm 0.01 \mathrm{~g}$ & na \\
\hline & HAE-EA & $8.48 \pm 0.21^{\mathrm{ab}}$ & $2.97 \pm 0.29 \mathrm{abc}$ & $100.94 \pm 1.41^{\mathrm{e}}$ & $0.86 \pm 0.04^{c}$ & $20.01 \pm 0.08^{b}$ \\
\hline & HAE-MEOH & $8.78 \pm 0.07^{\mathrm{a}}$ & na & $142.59 \pm 1.14^{\mathrm{d}}$ & $1.24 \pm 0.01^{\mathrm{a}}$ & $21.17 \pm 0.02^{\mathrm{a}}$ \\
\hline & Maceration EA & $8.35 \pm 0.20^{\mathrm{ab}}$ & $2.19 \pm 0.48^{c}$ & $94.19 \pm 0.88^{\mathrm{fg}}$ & $0.76 \pm 0.02$ de & $20.01 \pm 0.24^{b}$ \\
\hline & Maceration-MEOH & $8.62 \pm 0.04^{\mathrm{a}}$ & na & $146.76 \pm 2.41 \mathrm{bc}$ & $1.14 \pm 0.01^{b}$ & $21.17 \pm 0.11^{\mathrm{a}}$ \\
\hline & Maceration (not stirred)-EA & $8.56 \pm 0.23^{a}$ & $3.50 \pm 0.43^{\mathrm{a}}$ & $96.38 \pm 1.56^{\mathrm{fg}}$ & $0.71 \pm 0.02 \mathrm{e}$ & $0.96 \pm 0.19^{\mathrm{d}}$ \\
\hline & Maceration (not stirred)-MEOH & $8.56 \pm 0.08^{a}$ & na & $146.06 \pm 1.34 \mathrm{~cd}$ & $1.22 \pm 0.03^{\mathrm{a}}$ & $20.98 \pm 0.06^{\mathrm{a}}$ \\
\hline \multirow{7}{*}{ Bark } & infusion & $7.07 \pm 0.11^{\mathrm{c}}$ & na & $25.64 \pm 1.13^{h}$ & $0.42 \pm 0.04^{\mathrm{f}}$ & na \\
\hline & HAE-EA & $8.34 \pm 0.41^{\mathrm{ab}}$ & $2.52 \pm 0.41 \mathrm{bc}$ & $96.74 \pm 0.95^{f}$ & $0.82 \pm 0.01^{\mathrm{cd}}$ & $20.04 \pm 0.15^{b}$ \\
\hline & HAE-MEOH & $8.76 \pm 0.05^{\mathrm{a}}$ & $0.59 \pm 0.10$ de & $150.75 \pm 2.26^{\mathrm{ab}}$ & $1.27 \pm 0.02^{\mathrm{a}}$ & na \\
\hline & Maceration EA & $8.61 \pm 0.16^{\mathrm{a}}$ & $3.03 \pm 0.38^{a b}$ & $104.13 \pm 0.59^{\mathrm{e}}$ & $0.69 \pm 0.02 \mathrm{e}$ & $19.87 \pm 0.02^{b}$ \\
\hline & Maceration-MEOH & $8.76 \pm 0.04^{\mathrm{a}}$ & $0.52 \pm 0.03$ de & $154.33 \pm 1.10^{\mathrm{a}}$ & $1.23 \pm 0.01^{\mathrm{a}}$ & na \\
\hline & Maceration (not stirred)-EA & $8.04 \pm 0.14^{b}$ & $2.19 \pm 0.42^{c}$ & $92.22 \pm 0.61 \mathrm{~g}$ & $0.75 \pm 0.04$ de & $18.48 \pm 0.40^{\mathrm{c}}$ \\
\hline & Maceration (not stirred)-MEOH & $8.67 \pm 0.01^{\mathrm{a}}$ & $1.19 \pm 0.27^{\mathrm{d}}$ & $149.60 \pm 1.98 \mathrm{bc}$ & $1.19 \pm 0.03^{\mathrm{ab}}$ & na \\
\hline
\end{tabular}

* Values are reported as mean \pm S.D. GALAE: Galantamine equivalent; KAE: Kojic acid equivalent; ACAE: Acarbose equivalent; na: not active; HAE: Homogenizer assisted extraction; EA: Ethyl acetate; MEOH: Methanol. Different letters $(\mathrm{a}-\mathrm{g})$ indicate significant differences in the extracts $(p<0.05)$. 
Based on the results displayed in Table 3, it can be noticed that methanolic leaf and bark extracts (both prepared by HAE) exhibited relatively the same total antioxidant capacity ( 4.82 and $4.74 \mathrm{mmol} / \mathrm{TE}$, respectively) followed by methanolic leaf and bark (prepared by maceration) (4.29 and $4.51 \mathrm{mmol} / \mathrm{TE}$, respectively). Remarkable reducing potential was demonstrated by methanolic bark (HAE) (CUPRAC: 1129.33 and FRAP: $633.53 \mathrm{mg}$ TE/g) followed by methanolic leaf(HAE) (CUPRAC: 1047.10 and FRAP: $544.76 \mathrm{mg} \mathrm{TE} / \mathrm{g}$ ). However, lower activities were reported with the ethyl acetate leaf and bark extracts prepared by maceration (without stirring).

Chelation of transition metals is also considered as an evaluation of the antioxidant ability of a compound. Several lines of evidence reported that transition metals namely $\mathrm{Fe}^{2+}$ or $\mathrm{Cu}^{2+}$ are active redox metals and through Haber Weiss and Fenton reactions lead to the formation of ROS [22]. Evaluating the different extracts of $R$. racemosa showed us that the ethyl acetate leaves, and bark extracts prepared by maceration (without stirring) possessed the highest chelating power (29.39 and $24.93 \mathrm{mg}$ EDTAE/g, respectively).

\subsection{Enzymatic Inhibitory Effects}

The results of enzyme inhibitory effects are given in Table 4.

Furthermore, the extracts of $R$. racemos $a$ was screened for $\alpha$-amylase and $\alpha$-glucosidase inhibitory activities since diabetes mellitus type II (DMII) is a growing pandemic and people suffering from DMII has an increased risk in developing chronic macrovascular (cardiovascular disease) and microvascular (chronic kidney disease) complications [24]. Herein, the methanolic leaf and bark extracts (both prepared by HAE) exhibited the highest $\alpha$-amylase inhibitory activity (1.24 and $1.27 \mathrm{mmol} \mathrm{ACAE} / \mathrm{g}$, respectively). However, a different trend was observed with $\alpha$-glucosidase. For instance, both methanolic leaf extracts prepared by HAE and maceration significantly depressed $\alpha$-glucosidase activity (21.17 mmol ACAE/g, respectively). However, several extracts especially bark extracts were reported inactivity against $\alpha$-glucosidase.

\subsection{Exploratory Analysis, Classification and Discriminant Analysis}

After the initial screening of antioxidant properties and enzymatic inhibitory effects in the different $R$. racemosa extracts, multivariate statistics were employed on the dataset to group and then discriminate samples. Firstly the exploratory analysis i.e., PCA was computed and results were reported in Figure 1. The initial 11 biological parameters had generated three principal components, representing $92.3 \%$ of the total variance; in consideration of the eigenvalues which were larger than 1 (Figure 1A). The PC1 was much positively described with FRAP, CUPRAC, DPPH, ABTS and PPBD and its total variance was $60.5 \%$ (Figure $1 \mathrm{~B}$ ). This reflects strong properties in the anti-oxidants of samples being the right side of PC1, including Mac-MeOH, HAE-MeOH and Mac-(no stirring)-MeOH extracts of both organs. PC2 accounted for $22 \%$ of the total variance, exhibited significant positive loadings of AChE, glucosidase and tyrosinase. This indicates that PC2 separated, on its positive side, the samples showing excellent inhibition against $\mathrm{AChE}$, glucosidase and tyrosinase of those with lower inhibition activity on its negative side. Thus, ethyl acetate and methanolic extracts of leaves and stem bark obtained with maceration, homogenization and maceration (no stirring) exhibited better inhibition against AChE and tyrosinase. However, concerning glucosidase, better inhibition was achieved by the samples of both organs being of the left side of PC2 i.e., the ethyl acetate extracts obtained with maceration, homogenization and maceration (no stirring). The third component PC3, which summarized $9.8 \%$ of the data variance and discriminated samples according to their ability to chelate ferrous ion. Indeed the methanol extracts of leaves and stem bark derived from maceration (no stirring) showed a better ferrous ion chelating activity. Scores plots of the first three PC were depicted in Figure 1C; some groups can be visually distinguished. In particular, the methanol extracts of bark obtained with maceration (no stirring), maceration and homogenization were closed together. It was the same with the methanol extracts of leaves derived from homogenization and maceration (no stirring). Additionally, ethyl 
acetate extracts of both organs obtained using homogenization and maceration seemed to constitute the same group.

PCA allowed for the highlighting of a similarity in biological activities among several samples of R. racemosa. However, some samples could not be assigned to a certain well-defined group. For this purpose, the k-medoids clustering statistic was employed under the result of PCA for classification of samples into a small number of homogeneous clusters. From the cluster map reported in Figure 2A, it can be seen that the samples were partitioned in eight clusters. Indeed, besides the three clusters emphasized through PCA, five other clusters were identified. The quality of the clustering was assessed using the average silhouette approach. As observed (Figure 2B), the clustering was excellent with average silhouette width of 0.71 which had revealed close to 1 .

Unsupervised multivariate analysis helped to spotlight a great variation among samples, which were consolidated into eight distinct clusters. However, an in-depth analysis provided a view of the aggregation of some samples according to the organs and/or the solvents/techniques of extraction. The extracts of the two organs had partnered in a distinct cluster, except their extracts obtained with HAE-EA and Mac-EA. In addition, while in the bark, Mac-MeOH, HAE-MeOH and Mac-(no stirring)-MeOH extracts were clustered together, in leaves, $\mathrm{Mac}-\mathrm{MeOH}$ extracts differed from those of HAE-MeOH and Mac-(no stirring)-MeOH. This remark led us to successively achieved a bark and leaves discrimination and to assess the impact of the nature of techniques of extraction on their respective biological activities.

For tentative diagnosing discrimination between the two organs, datasets derived from evaluated biological activities were submitted to PLS-discriminant analysis. Scatter plot of PLS-DA analysis, pointed to a separation of bark and leaves extracts, with satisfactory parameters, being 0.77 for AUC when taking into account the first two components (Figure 3A,B). Afterwards, the most significant assays that allowed us to separate the two organs were identified. Among all evaluated biological activities glucosidase, MCA and DPPH were found to be the most important assays for organ discrimination (Figure 3C). A comparative assessment into the consideration of these biological assays showed that the highest DPPH radical scavenging and $\mathrm{Cu}^{2+}$ chelating activity was observed in the stem bark samples while leaf samples exhibited important inhibitory against glucosidase compare to those of stem bark (Figure 4).

Considering the great variations among the samples emphasized by multivariate statistics, it is important to note that the phytoconstituents recovery and in a wider the biological activities of $R$. racemosa were strongly impacted by the nature of solvents/technique of extraction used and varied greatly from one organ too another. In fact, various kinds of constituents, categorized into primary and secondary metabolites, are synthesized by the plant. Secondary metabolites such as phenolics, play a crucial role in plant functions and the interaction between plant and its environment. Further, the distribution and accumulation of secondary metabolites within the different organs, tissues and cells of plants, depend on their specific biological functions. As a result, the choice of solvent and technique for herbals phytoconstituents extraction should take into account their chemical characteristics, polarities and uneven distribution in the herbals matrix. 


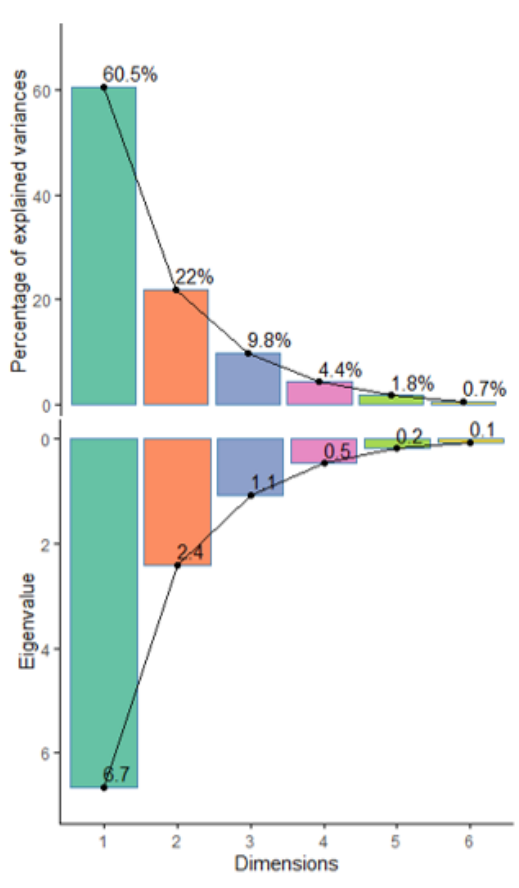

A

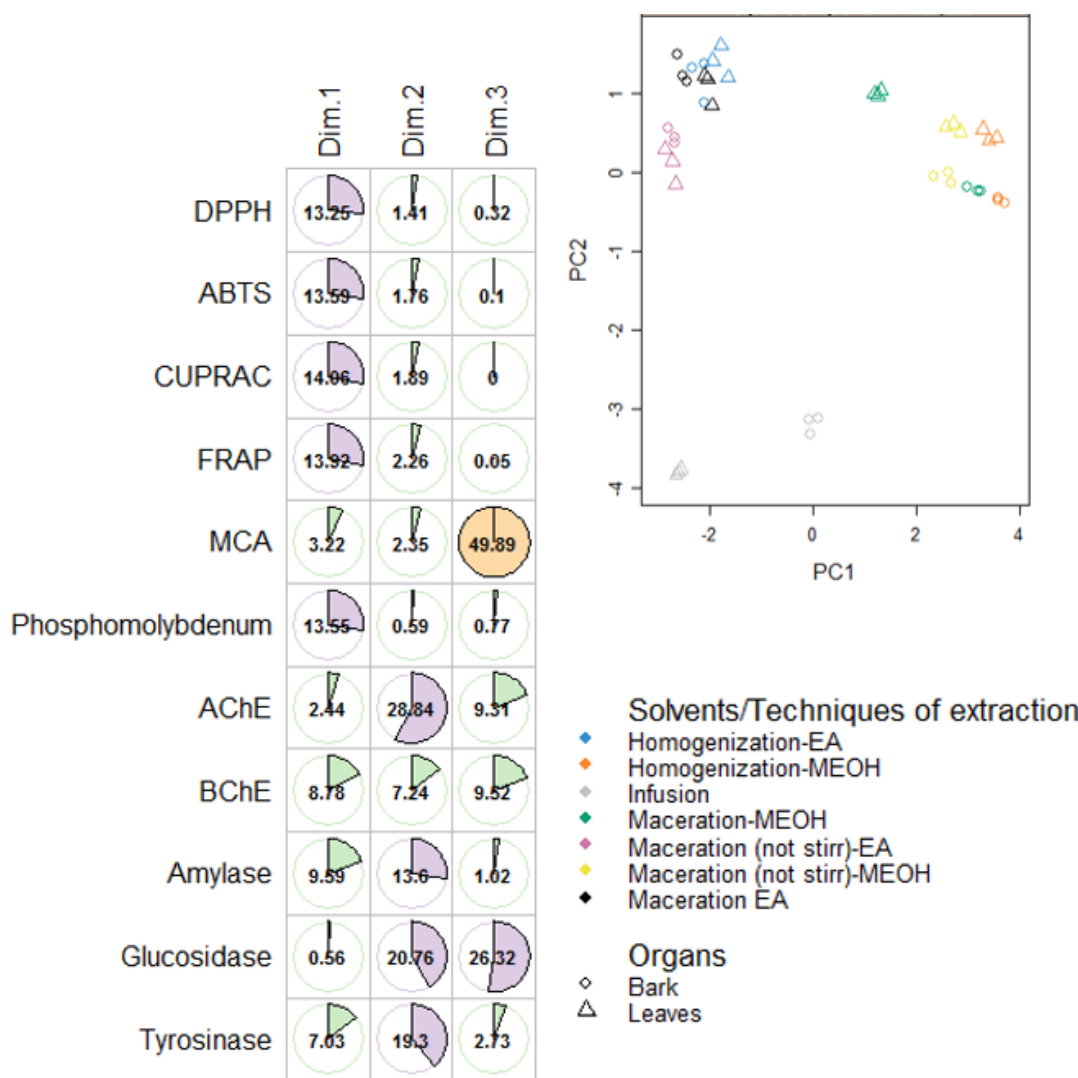

B
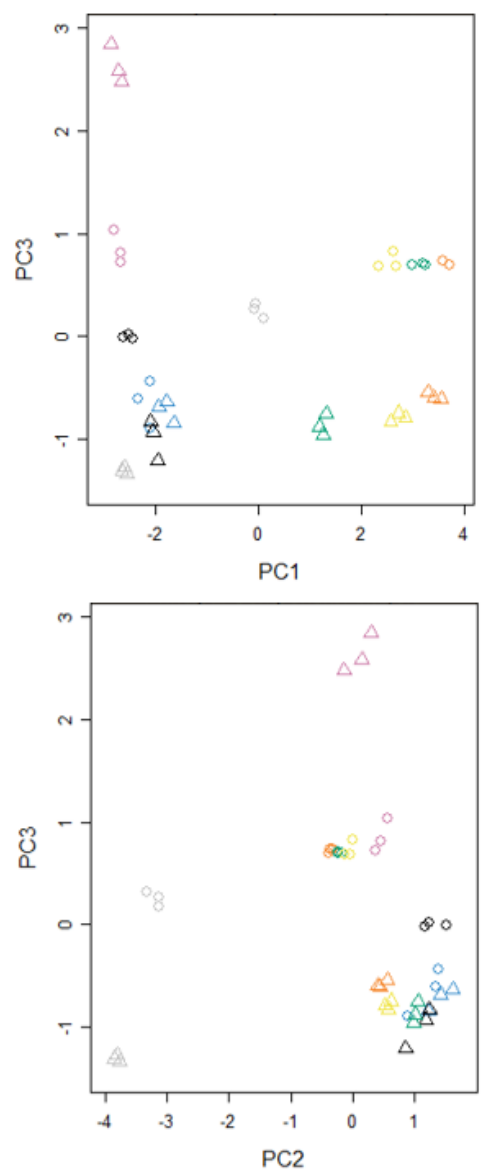

Figure 1. Principal component analysis on R. racemose biological activities. (A). Percentage of variability and eigenvalue explained by each principal component. (B). Relation between the biological activities and the first three principal components. (C). Samples plots. 



Figure 2. Cluster plot (A) and Silhouette plot (B) of K-medoids analysis. 
$\mathbf{A}$
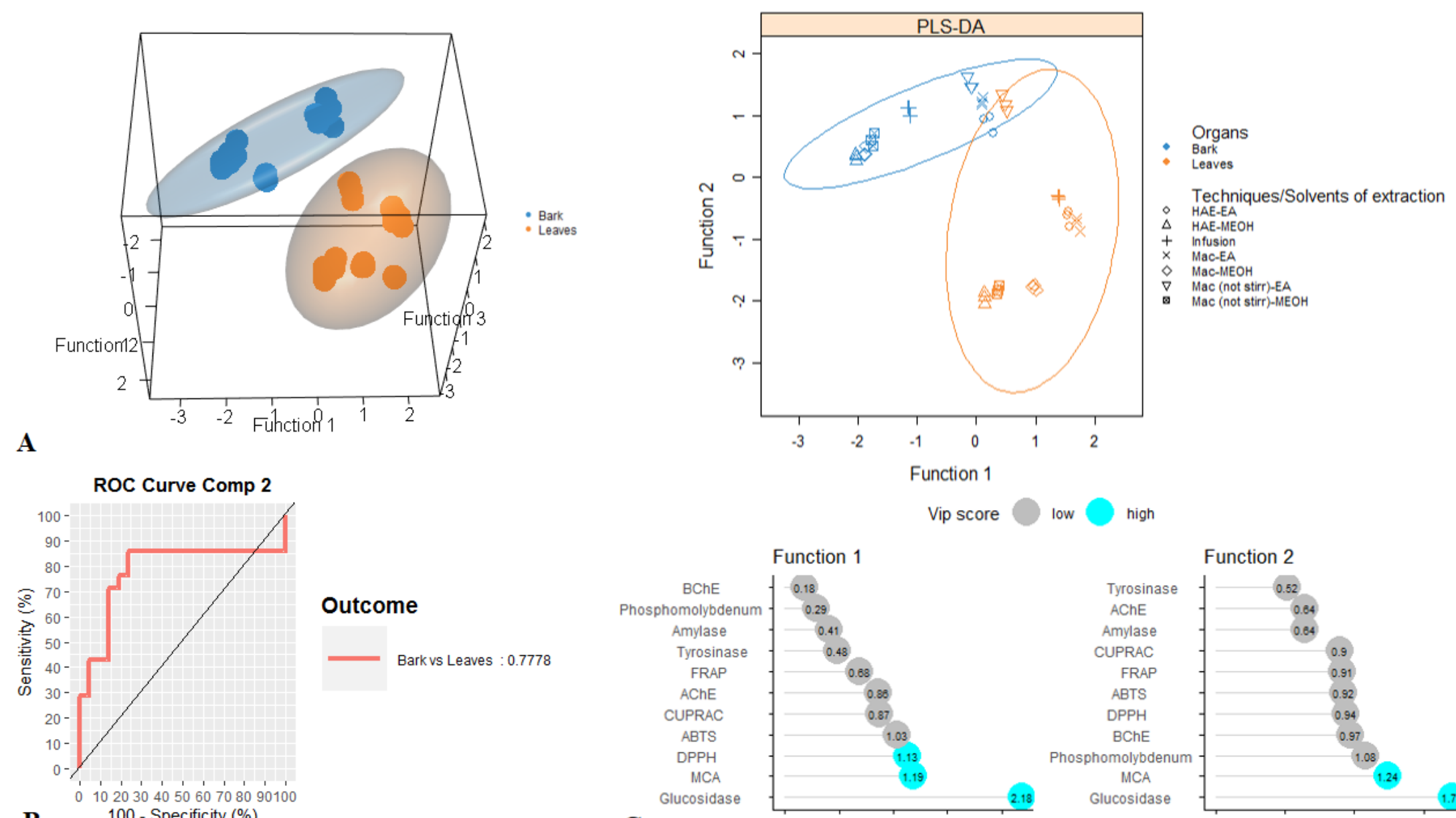

B

100 - Specificity $(\%)$
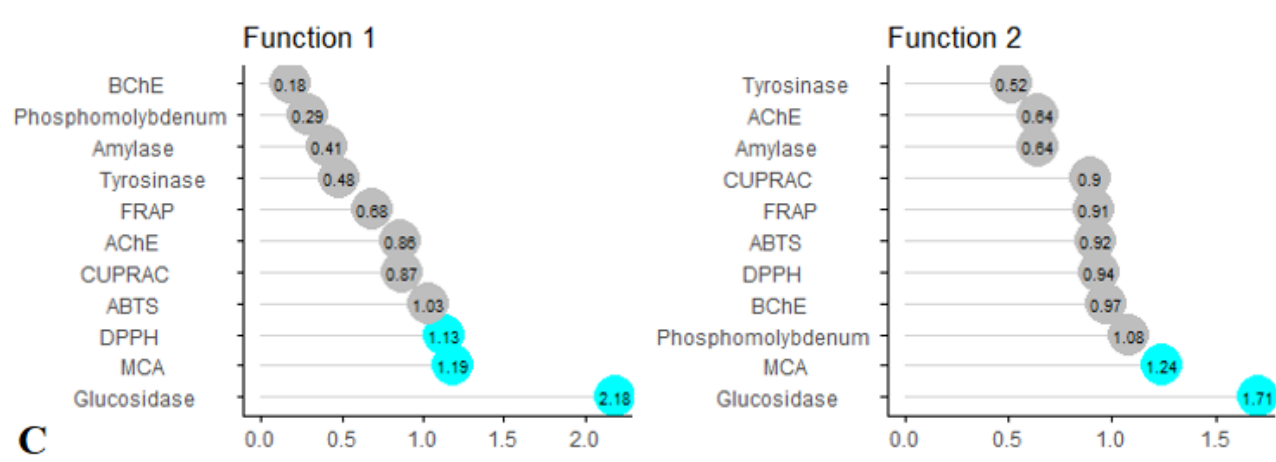

Figure 3. Discrimination between leaves and bark samples through PLS-DA. (A). Three- and two-dimensional score plots. (B). Area Under the Curve average using one-vs-all comparisons. (C). Biological activities assays explaining the largest part of the variance between the two organs. 

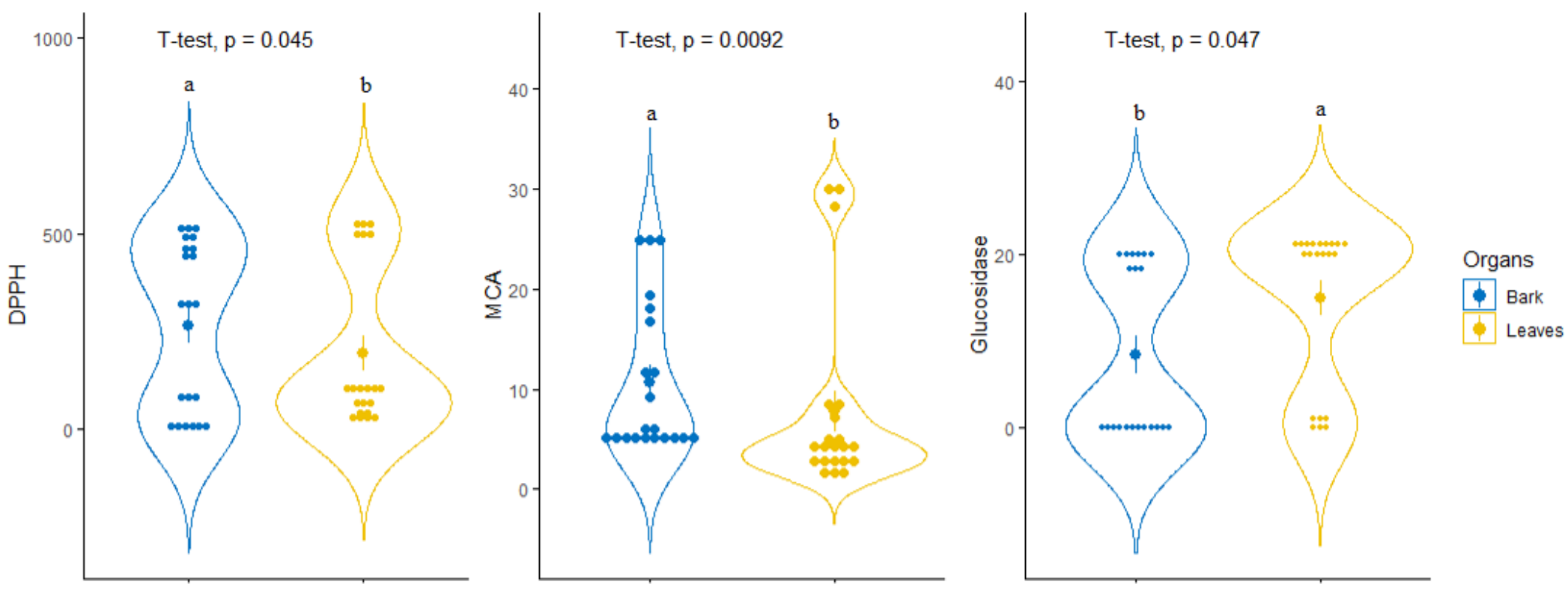

Figure 4. Comparison of leaves and bark taking account of the identified most discriminant biological activities. 


\subsection{Anti-bacterial and Anti-fungal Activities}

All extracts showed anti-microbial activity within the concentration range tested (Table 5) but with wide variability in terms of potency and selectivity. The strongest inhibition was observed for leaf extracts obtained by static maceration in EA and $\mathrm{MeOH}$ (MIC $0.9 \mathrm{mg} / \mathrm{mL}$, against E. coli) while the extract obtained by maceration of bark in methanol resulted in reducing $P$. aeruginosa growth. The differences between the activity of extracts from different plant parts do not highlight significant differences, as evidenced by the similar profile for the water infusion, $\mathrm{HAE}-\mathrm{MeOH}$ and static maceration in methanol that could be considered as the extracts with a broader spectrum of activity, with MIC values lower than $1 \mathrm{mg} / \mathrm{mL}$ on all tested strains. Conversely, the EA extraction by static maceration seems to be ineffective against all tested microorganisms. Among leaf extracts, the HAE-EA results were the most promising in terms of efficacy and spectrum of activity. All strains are sensible with MIC values lower than $0.37 \mathrm{mg} / \mathrm{mL}$. The same extracts obtained from bark give similar results, but with slightly higher MIC values against E. coli and S. aureus $(0.56 \mathrm{mg} / \mathrm{mL})$.

Table 5. Antibacterial activity of tested samples, results are in $\mathrm{mg} / \mathrm{mL}$.

\begin{tabular}{|c|c|c|c|c|c|c|c|c|}
\hline Samples & MIC/MBC & S.a. & B.c. & L.m. & P.a. & E.c. & S.t. & En.cl. \\
\hline \multirow{2}{*}{ Leaves- infusion } & MIC & 0.75 & 0.37 & 0.37 & 0.30 & 0.43 & 0.37 & 0.56 \\
\hline & MBC & 1.50 & 0.75 & 0.75 & 0.43 & 0.87 & 0.75 & 0.75 \\
\hline \multirow{2}{*}{ Leaves- HAE-EA } & MIC & 0.75 & 0.75 & 1.12 & 0.43 & 0.30 & 0.56 & 0.75 \\
\hline & $\mathrm{MBC}$ & 1.50 & 1.50 & 1.50 & 0.87 & 0.43 & 0.75 & 1.50 \\
\hline \multirow{2}{*}{ Leaves- HAE-MEOH } & MIC & 0.37 & 0.13 & 0.27 & 0.27 & 0.27 & 0.27 & 0.37 \\
\hline & $\mathrm{MBC}$ & 0.75 & 0.18 & 0.37 & 0.37 & 0.37 & 0.37 & 0.75 \\
\hline \multirow{2}{*}{ Leaves- Maceration EA } & MIC & 1.12 & 1.12 & 1.12 & 0.30 & 0.43 & 1.12 & 1.12 \\
\hline & MBC & 1.50 & 1.50 & 1.50 & 0.43 & 0.87 & 1.50 & 1.50 \\
\hline \multirow{2}{*}{ Leaves- Maceration-MEOH } & MIC & 0.37 & 0.27 & 0.56 & 0.27 & 0.18 & 0.56 & 0.56 \\
\hline & $\mathrm{MBC}$ & 0.75 & 0.37 & 0.75 & 0.37 & 0.37 & 0.75 & 0.75 \\
\hline \multirow{2}{*}{ Leaves- Maceration (not stirred)-EA } & MIC & 1.50 & 0.75 & 1.12 & 0.18 & 0.09 & 0.75 & 1.12 \\
\hline & $\mathrm{MBC}$ & 3.00 & 1.50 & 1.50 & 0.37 & 0.18 & 1.50 & 1.50 \\
\hline \multirow{2}{*}{ Leaves- Maceration (not stirred)-MEOH } & MIC & 0.37 & 0.27 & 0.56 & 0.37 & 0.09 & 0.56 & 0.56 \\
\hline & $\mathrm{MBC}$ & 0.75 & 0.37 & 0.75 & 0.75 & 0.18 & 0.75 & 0.75 \\
\hline \multirow{2}{*}{ Bark- infusion } & MIC & 0.56 & 0.27 & 0.75 & 0.75 & 0.18 & 0.37 & 0.37 \\
\hline & $\mathrm{MBC}$ & 0.75 & 0.37 & 1.50 & 1.50 & 0.37 & 0.75 & 0.75 \\
\hline \multirow{2}{*}{ Bark- HAE-EA } & MIC & 0.75 & 0.56 & 0.75 & 0.65 & 0.75 & 0.75 & 1.50 \\
\hline & $\mathrm{MBC}$ & 1.50 & 0.75 & 1.50 & 0.87 & 1.50 & 1.50 & 3.00 \\
\hline \multirow{2}{*}{ Bark- HAE-MEOH } & MIC & 0.56 & 0.18 & 0.37 & 0.37 & 0.56 & 0.37 & 0.37 \\
\hline & $\mathrm{MBC}$ & 0.75 & 0.37 & 0.75 & 0.75 & 0.75 & 0.75 & 0.75 \\
\hline \multirow{2}{*}{ Bark- Maceration EA } & MIC & 0.56 & 0.18 & 0.75 & 0.75 & 0.56 & 1.12 & 0.75 \\
\hline & $\mathrm{MBC}$ & 0.75 & 0.37 & 1.5 & 1.50 & 0.75 & 1.50 & 1.50 \\
\hline \multirow{2}{*}{ Bark- Maceration-MEOH } & MIC & 0.37 & 0.27 & 0.75 & 0.75 & 0.27 & 1.12 & 1.12 \\
\hline & $\mathrm{MBC}$ & 0.75 & 0.37 & 1.50 & 1.50 & 0.37 & 1.50 & 1.50 \\
\hline \multirow{2}{*}{ Bark- Maceration (not stirred)-EA } & MIC & 2.25 & 2.25 & 1.50 & 1.50 & 2.25 & 1.12 & 1.12 \\
\hline & $\mathrm{MBC}$ & 3.00 & 3.00 & 3.00 & 3.00 & 3.00 & 1.50 & 1.50 \\
\hline \multirow{2}{*}{ Bark- Maceration (not stirred)-MEOH } & MIC & 0.27 & 0.27 & 0.56 & 0.09 & 0.56 & 0.56 & 0.37 \\
\hline & $\mathrm{MBC}$ & 0.37 & 0.37 & 0.75 & 0.18 & 0.75 & 0.75 & 0.75 \\
\hline \multirow{2}{*}{ Streptomycin } & MIC & 0.10 & 0.025 & 0.15 & 0.025 & 0.10 & 0.10 & 0.025 \\
\hline & MBC & 0.20 & 0.05 & 0.30 & 0.05 & 0.20 & 0.20 & 0.05 \\
\hline \multirow{2}{*}{ Ampicillin } & MIC & 0.10 & 0.10 & 0.15 & 0.05 & 0.15 & 0.10 & 0.10 \\
\hline & $\mathrm{MBC}$ & 0.15 & 0.15 & 0.30 & 0.10 & 0.20 & 0.20 & 0.15 \\
\hline
\end{tabular}

Escherichia coli (E.c.), Pseudomonas aeruginosa (P.a.), Salmonella typhimurium (S.t.), Listeria monocytogenes (L.m.), Enterobacter cloacae (E.c.), Bacillus cereus (B.c.), Micrococcus luteus (M.l.), Enterobacter cloacae (En.cl.) and Staphylococcus aureus (S.a.).

All tested extracts resulted active as inhibitory of fungal growth, but huge variability was recorded between the most active (bark HAE-MEOH on T. viride: MIC $0.004 \mathrm{mg} / \mathrm{mL}$ ) and the less active (bark HAE-EA on A. fumigatus: MIC $3.0 \mathrm{mg} / \mathrm{mL}$ ). The strongest activity was registered for bark extracts HAE-MEOH and maceration-MEOH against T. viride with MIC values of $0.004 \mathrm{mg} / \mathrm{mL}$ and $0.017 \mathrm{mg} / \mathrm{mL}$, respectively. The less sensible strain resulted A. fumigatus (MIC range 3-0.56 mg/mL) while all extracts actively reduce the viability of $T$. viride in the concentration range $0.004-0.75 \mathrm{mg} / \mathrm{mL}$. Of the 14 tested 
extracts, 9 were able to inhibit the fungal growth at concentration $0.37 \mathrm{mg} / \mathrm{mL}$ or lower. Leaf maceration in not stirred $\mathrm{MEOH}$ and infusion are the extracts with a broader spectrum of activity, they inhibit the growth of all tested strains at low concentrations (MIC range $0.75-0.13 \mathrm{mg} / \mathrm{mL}$ and $0.56-0.09 \mathrm{mg} / \mathrm{mL}$, respectively, with the only exception of higher MIC value registered for infusion on the less sensible A. fumigatus (Table 6). Conversely, the least promising extract as anti-fungal agents in bark HAE-EA that showed inhibition values higher than 1.5 except for the value registered against $T$ viride. The MIC of $0.56 \mathrm{mg} / \mathrm{mL}$ results anyway one of the higher against this strain. The activity of leaves extracts Maceration-MEOH against $A$. ochraceus (MIC $0.18 \mathrm{mg} / \mathrm{mL}$ ) and maceration (not stirred)-MEOH on P. ochrochlorum (MIC $0.13 \mathrm{mg} / \mathrm{mL}$ ) was also notable. No general consideration is highlighted in terms of anti-fungal potency comparing the extracts from bark or leaves. Considering a cutoff at $0.75 \mathrm{mg} \mathrm{mL}^{-1}$ as an indicator for relevant anti-fungal activity, the efficacy scale, based on the number of sensible strains results leaves infusion and maceration (not stirred)-MEOH $>$ bark maceration (not stirred)-EA and maceration (not stirred)-MeOH $>$ bark HAE-MEOH $>$ bark Bark-maceration-MEOH, leaves maceration-MEOH and HAE-MEOH $>$ leaves maceration EA $>$ bark infusion and maceration EA, leaves HAE-EA and maceration (not stirred)-EA > bark HAE-EA.

Table 6. Anti-fungal activity of tested samples, results are in $\mathrm{mg} / \mathrm{mL}$.

\begin{tabular}{|c|c|c|c|c|c|c|c|c|c|}
\hline Samples & MIC/MFC & A.f. & A.o. & A.n. & A.v. & P.o. & P.f. & T.v. & P.v.c. \\
\hline \multirow{2}{*}{ Leaves- infusion } & MIC & 2.25 & 0.37 & 0.37 & 0.37 & 0.09 & 0.18 & 0.27 & 0.56 \\
\hline & MFC & 3.00 & 0.75 & 0.75 & 0.75 & 0.18 & 0.37 & 0.37 & 0.75 \\
\hline \multirow{2}{*}{ Leaves- HAE-EA } & MIC & 1.12 & 0.75 & 1.50 & 0.75 & 0.37 & 0.75 & 0.75 & 1.50 \\
\hline & MFC & 1.50 & 1.50 & 3.00 & 1.50 & 0.75 & 1.50 & 1.50 & 3.00 \\
\hline \multirow{2}{*}{ Leaves- HAE-MEOH } & MIC & 0.56 & 0.75 & 0.75 & 0.27 & 1.12 & 0.37 & 0.22 & 0.75 \\
\hline & MFC & 1.12 & 1.50 & 1.50 & 0.37 & 1.50 & 0.75 & 0.44 & 1.50 \\
\hline \multirow{2}{*}{ Leaves- Maceration EA } & MIC & 1.50 & 0.37 & 1.50 & 0.75 & 0.37 & 1.12 & 0.37 & 1.50 \\
\hline & MFC & 3.00 & 0.75 & 3.00 & 1.50 & 0.75 & 1.50 & 0.75 & 3.00 \\
\hline \multirow{2}{*}{ Leaves- Maceration-MEOH } & MIC & 0.75 & 0.18 & 0.75 & 0.37 & 0.75 & 0.37 & 0.22 & 0.75 \\
\hline & MFC & 1.50 & 0.37 & 1.50 & 0.75 & 1.50 & 0.75 & 0.44 & 1.50 \\
\hline \multirow{2}{*}{ Leaves- Maceration (not stirred)-EA } & MIC & 1.50 & 0.75 & 1.50 & 0.37 & 1.12 & 0.75 & 0.37 & 1.12 \\
\hline & MFC & 3.00 & 1.50 & 3.00 & 0.75 & 1.50 & 1.50 & 0.75 & 1.50 \\
\hline \multirow{2}{*}{$\begin{array}{l}\text { Leaves- Maceration (not } \\
\text { stirred)-MEOH }\end{array}$} & MIC & 0.75 & 0.18 & 0.27 & 0.37 & 0.13 & 0.27 & 0.37 & 0.37 \\
\hline & MFC & 1.50 & 0.37 & 0.37 & 0.75 & 0.18 & 0.37 & 0.75 & 0.75 \\
\hline \multirow{2}{*}{ Bark- infusion } & MIC & 0.75 & 0.37 & 0.75 & 1.12 & 1.12 & 0.75 & 0.27 & 0.84 \\
\hline & MFC & 1.50 & 0.75 & 1.50 & 1.50 & 1.15 & 1.50 & 0.37 & 1.12 \\
\hline \multirow{2}{*}{ Bark- HAE-EA } & MIC & 3.00 & 3.00 & 3.00 & 1.50 & 2.25 & 3.00 & 0.56 & 3.00 \\
\hline & MFC & 6.00 & 6.00 & 6.00 & 3.00 & 3.00 & 6.00 & 0.75 & 6.00 \\
\hline \multirow{2}{*}{ Bark- HAE-MEOH } & MIC & 0.75 & 0.37 & 0.75 & 1.50 & 0.75 & 0.37 & 0.004 & 0.56 \\
\hline & MFC & 1.50 & 0.75 & 1.50 & 3.00 & 1.50 & 0.75 & 0.008 & 0.75 \\
\hline \multirow{2}{*}{ Bark- Maceration EA } & MIC & 1.12 & 0.37 & 0.75 & 0.75 & 0.75 & 1.12 & 0.27 & 0.75 \\
\hline & MFC & 1.50 & 0.75 & 1.50 & 1.50 & 1.50 & 1.50 & 0.37 & 1.50 \\
\hline \multirow{2}{*}{ Bark- Maceration-MEOH } & MIC & 1.12 & 0.37 & 0.75 & 0.75 & 0.27 & 0.27 & 0.017 & 1.12 \\
\hline & MFC & 1.50 & 0.75 & 1.50 & 1.50 & 0.37 & 0.37 & 0.025 & 1.15 \\
\hline \multirow{2}{*}{ Bark- Maceration (not stirred)-EA } & MIC & 0.75 & 0.37 & 0.56 & 0.37 & 0.56 & 0.56 & 0.27 & 0.75 \\
\hline & MFC & 1.50 & 0.75 & 0.75 & 0.75 & 0.75 & 0.75 & 0.37 & 1.50 \\
\hline \multirow{2}{*}{ Bark- Maceration (not stirred)-MEOH } & MIC & 0.75 & 0.37 & 0.56 & 0.37 & 0.56 & 0.37 & 0.18 & 1.12 \\
\hline & MFC & 1.50 & 0.75 & 0.75 & 0.75 & 0.75 & 1.50 & 0.37 & 1.15 \\
\hline \multirow{2}{*}{ Bifonazole } & MIC & 0.15 & 0.15 & 0.15 & 0.1 & 0.20 & 0.2 & 0.15 & 0.1 \\
\hline & MFC & 0.2 & 0.20 & 0.2 & 0.2 & 0.25 & 0.25 & 0.2 & 0.2 \\
\hline \multirow{2}{*}{ Ketoconazole } & MIC & 0.2 & 0.15 & 0.2 & 0.2 & 1.00 & 0.2 & 1 & 0.2 \\
\hline & MFC & 0.5 & 0.20 & 0.5 & 0.5 & 1.50 & 0.5 & 1.5 & 0.3 \\
\hline
\end{tabular}

Aspergillus fumigatus (A.f.), Aspergillus niger (A.n.), Trichoderma viride (T.v.), Penicillium funiculosum (P.f.), Penicillium ochrochloron (P.o) and Penicillium verrucosum (P.v.).

\subsection{Bioinformatics and Docking Studies}

Finally, based on both phytochemical and biological assays, a bioinformatics study was conducted to predict the pharmacokinetics and putative targets of phytochemicals identified in the extracts. The bioinformatics evaluation was performed using SwissADME and SwissTargetPrediction software (Swiss Institute of Bioinformatics) and complete results are reported as supplementary material 
(Supplementary Material_Bioinformatic Folder). Specifically, the evaluation considered more than 30 phytochemicals that were identified in all analyzed and tested extracts. As expected, a wide plethora of target proteins was predicted by SwissTargetPrediction software, although with different interaction probability (range: $0.05-1 \mathrm{mg} / \mathrm{mL}$ ). The phytocompounds were then classified according to their capability to be absorbed by the gastrointestinal tract (GI), to cross blood-brain barrier (BBB) and interact with the tested enzymes, namely cholinesterases, $\alpha$-glucosidase, $\alpha$-amylase and tyrosinase (Figure 5).

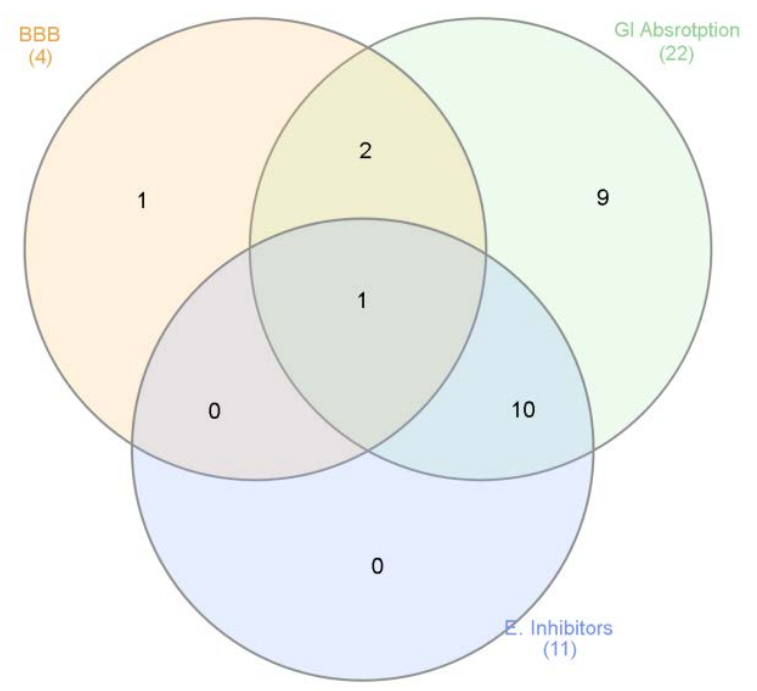

Figure 5. Venn diagram summarizing the criteria for selecting extracts' phytochemicals, basing on pharmacokinetics (Gastrointestinal (GI) absorption and blood brain barrier (BBB) crossing) and biological properties (enzyme inhibition).

Subsequently, a network pharmacology analysis was performed via Cytoscape software to highlight the most probable putative interactions, towards tested enzymes. Although no identified phytochemical was predicted to interact with $\alpha$-glucosidase and/or $\alpha$-amylase, thus indicating that the inhibition of these two enzymes could be aspecific, multiple secondary metabolites present in the extracts were predicted to interact with tyrosinase and cholinesterases. Regarding the cholinesterases, the interactions predicted for abscisic acid could be of interest and consistent with the ability of this compound to cross BBB. Conversely, the lack of capability to cross BBB displayed by apigenin, luteolin and quercetin scale back their importance as anti-cholinesterase agents (Supplementary Material_Bioinformatic Folder; Figure 6). Nevertheless, these compounds, but also vitexin, isovitexin, methoxy-trihydroxy(iso)flavone and procyanidin B, were predicted to interact with tyrosinase (Figure 6). Given the anti-tyrosinase effects of the tested extracts, the predicted interactions could be therapeutically relevant against hyperpigmentation. Furthermore, isovitexin, vitexin, apigenin, luteolin and quercetin were predicted to interact with metalloproteinase-12 (MMP-12) (Figure 6). whose activity was significantly increased in skin disorders, including melanoma [25].

Therefore, a docking analysis was carried out, to investigate the interactions between apigenin, quercetin, vitexin, isovitexin, methoxy-trihydroxy(iso)flavone and procyanidin B with tyrosinase and MMP-12 (Figures 7 and 8).

Furthermore, the tested extracts were also effective towards multiple bacterial and fungi strains involved in skin disorders [26-34], thus highlighting the importance to deepen our knowledge about the mechanisms underlying the aforementioned inhibitory effects. In this context, these compounds were docked towards DNA-gyrase and 14-lanosterol- $\alpha$-demethylase, which are key enzymes in the metabolism of bacteria and fungi, respectively $[35,36]$. The compounds were also docked against DNA-gyrase and 14-lanosterol- $\alpha$-dmethylase. The results of the docking study are reported in Figures 9 and 10 , including $\Delta \mathrm{G}$ and $\mathrm{Ki}$ values. 


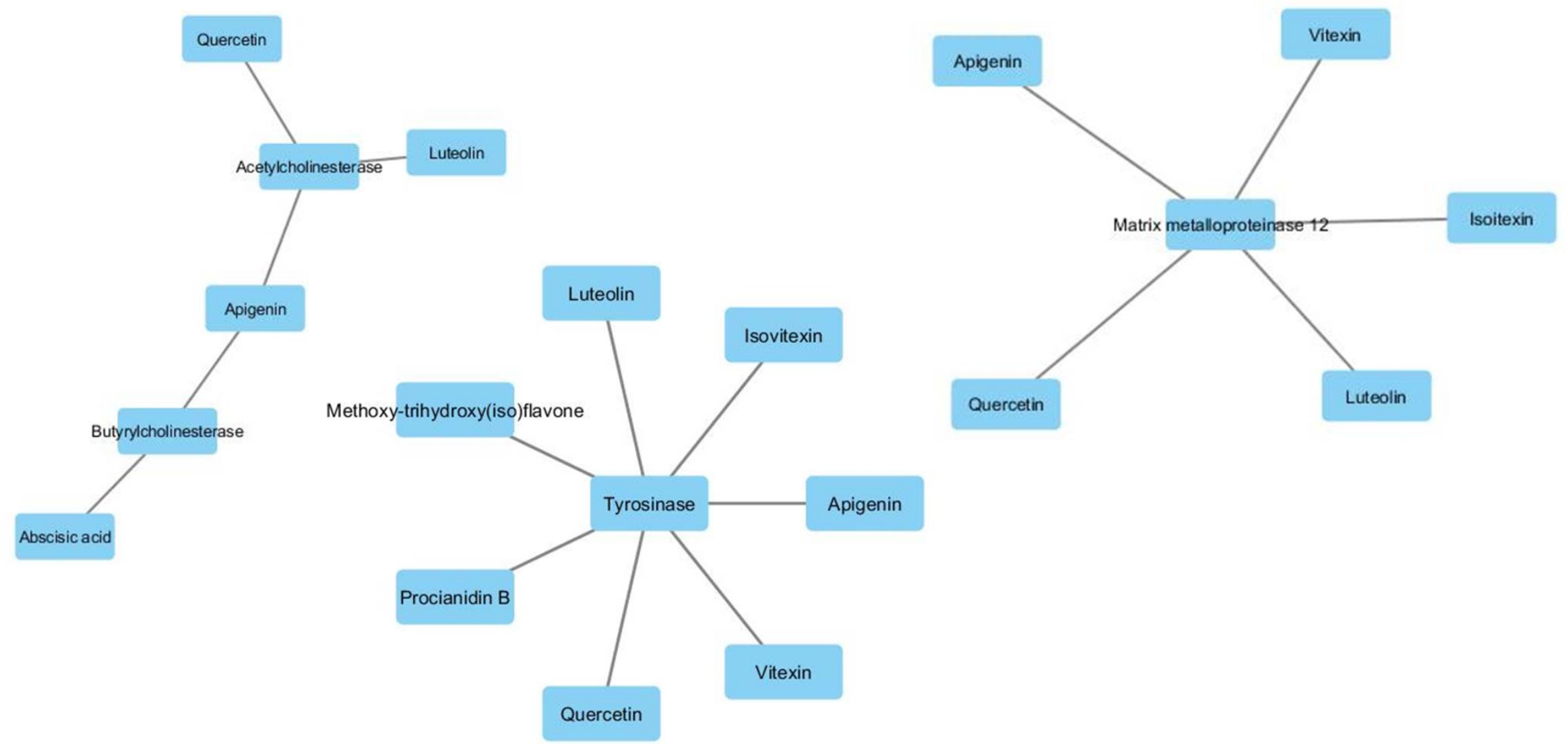

Figure 6. Components-targets analysis highlighting the putative interactions of luteolin, apigenin, vitexin, isovitexin, quercetin, procyanidin B and methoxy-trihydroxy(iso)flavone with tyrosinase, cholinesterases and metalloproteinase- 12 . 


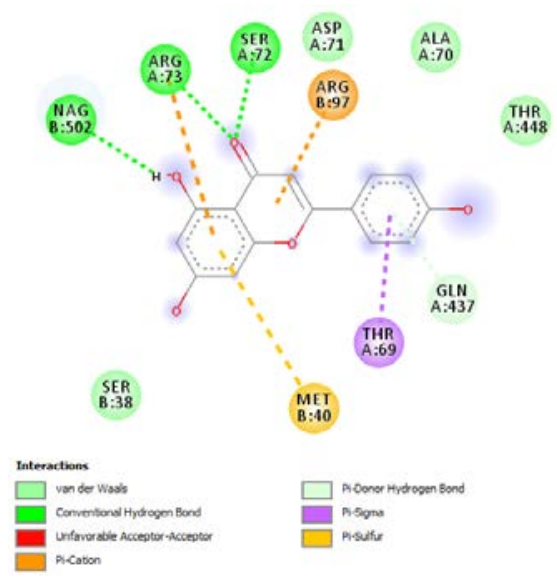

(a)
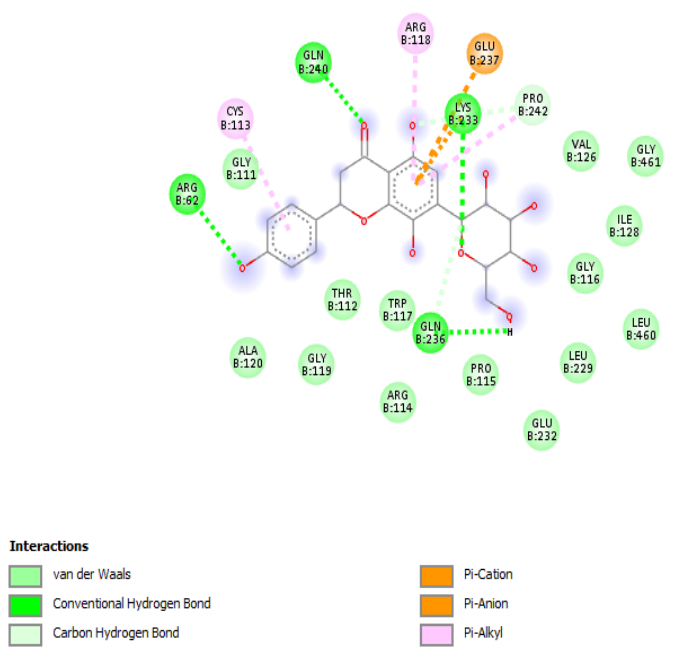

(c)

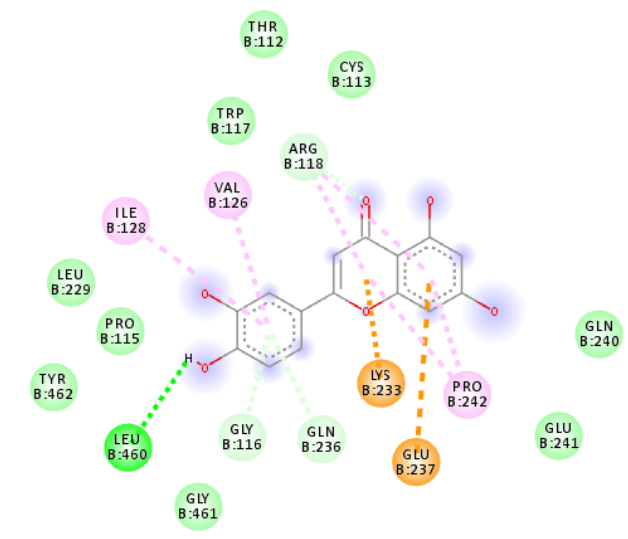

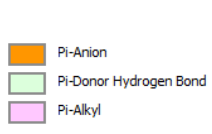

(e)

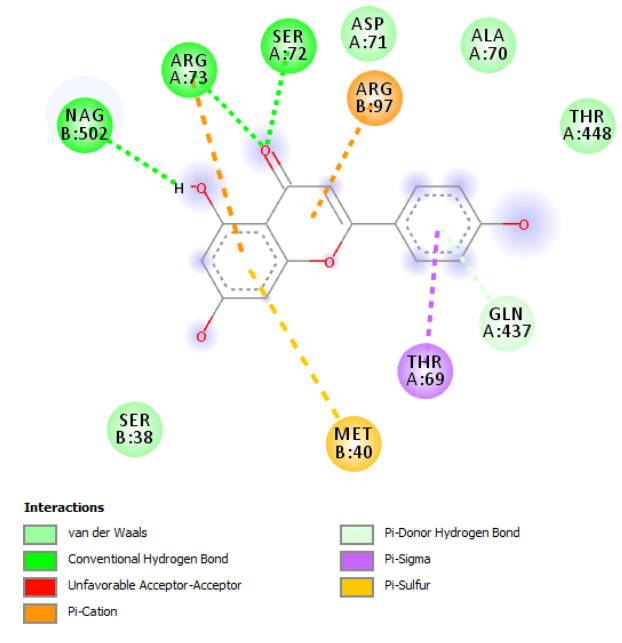

(b)

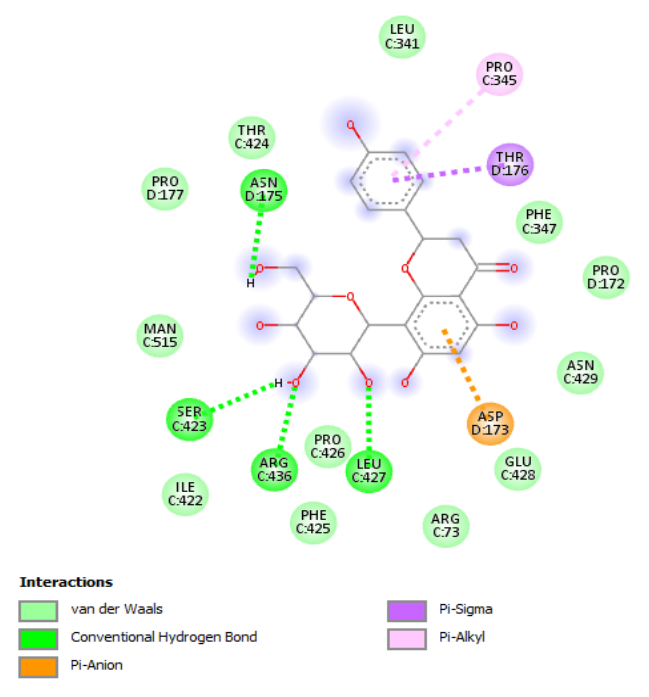

(d)
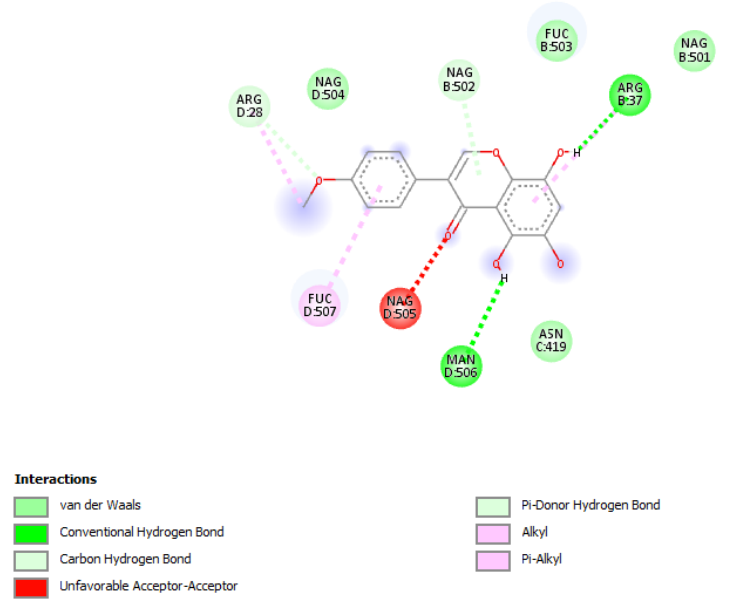

(f)

Figure 7. Cont. 


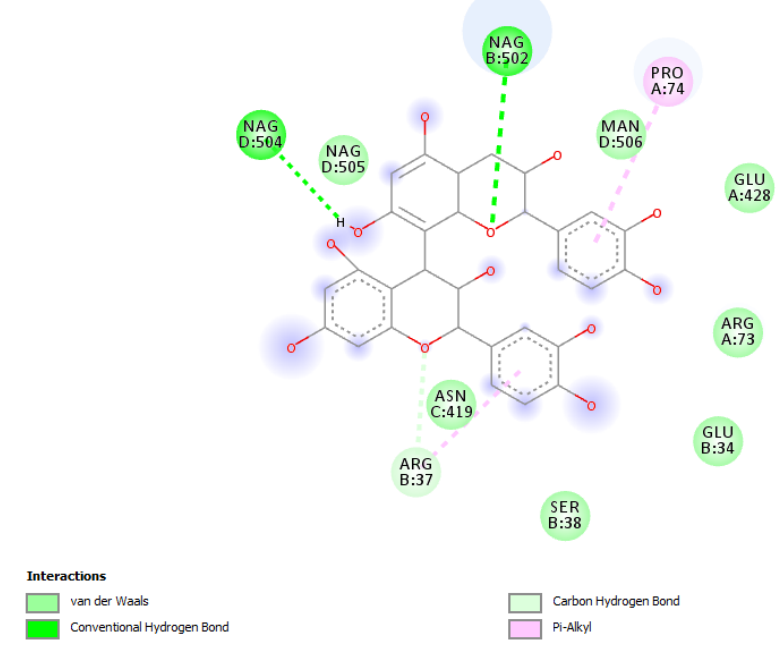

(g)

Figure 7. (a) Putative interactions between apigenin and tyrosinase (Protein Data Bank (PDB): 5M8P). Free energy of binding $(\Delta \mathrm{G})$ and affinity $(\mathrm{Ki})$ are $-6.8 \mathrm{kcal} / \mathrm{mol}$ and $10.5 \mu \mathrm{M}$, respectively. (b) Putative interactions between quercetin and tyrosinase (PDB: 5M8P). Free energy of binding $(\Delta \mathrm{G})$ and affinity $(\mathrm{Ki})$ are $-7.1 \mathrm{kcal} / \mathrm{mol}$ and $6.3 \mu \mathrm{M}$, respectively. (c) Putative interactions between isovitexin and tyrosinase (PDB: $5 \mathrm{M} 8 \mathrm{P}$ ). Free energy of binding $(\Delta \mathrm{G})$ and affinity $(\mathrm{Ki})$ are $-8.9 \mathrm{kcal} / \mathrm{mol}$ and $0.3 \mu \mathrm{M}$, respectively. (d) Putative interactions between vitexin and tyrosinase (PDB: 5M8P). Free energy of binding $(\Delta G)$ and affinity (Ki) are $-9.0 \mathrm{kcal} / \mathrm{mol}$ and $0.3 \mu \mathrm{M}$, respectively. (e) Putative interactions between luteolin and tyrosinase (PDB: 5M8P). Free energy of binding $(\Delta \mathrm{G})$ and affinity $(\mathrm{Ki})$ are $-8.3 \mathrm{kcal} / \mathrm{mol}$ and $0.8 \mu \mathrm{M}$, respectively. (f) Putative interactions between methoxy-trihydroxy(iso)flavone and tyrosinase (PDB: $5 \mathrm{M} 8 \mathrm{P})$. Free energy of binding $(\Delta \mathrm{G})$ and affinity $(\mathrm{Ki})$ are $-6.5 \mathrm{kcal} / \mathrm{mol}$ and $17.4 \mu \mathrm{M}$, respectively. $(\mathrm{g})$ Putative interactions between procyanidin B and tyrosinase (PDB: 5M8P). Free energy of binding $(\Delta \mathrm{G})$ and affinity $(\mathrm{Ki})$ are $-8.6 \mathrm{kcal} / \mathrm{mol}$ and $0.5 \mu \mathrm{M}$, respectively.

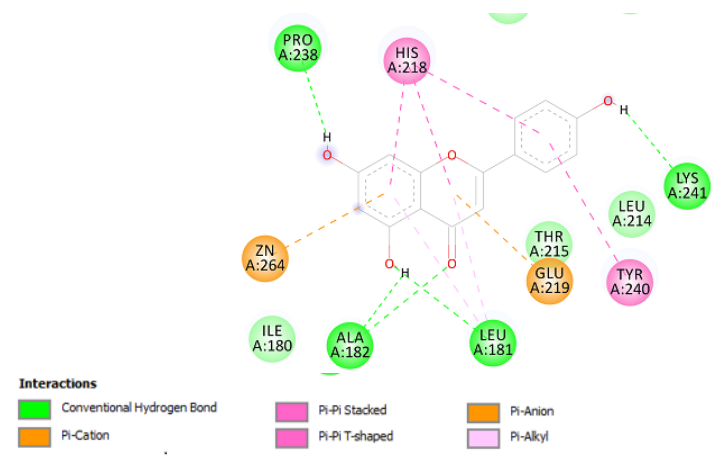

(a)

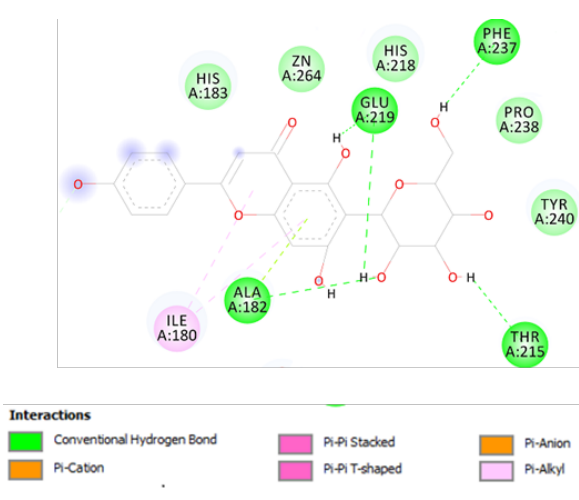

(b)

Figure 8. Cont. 


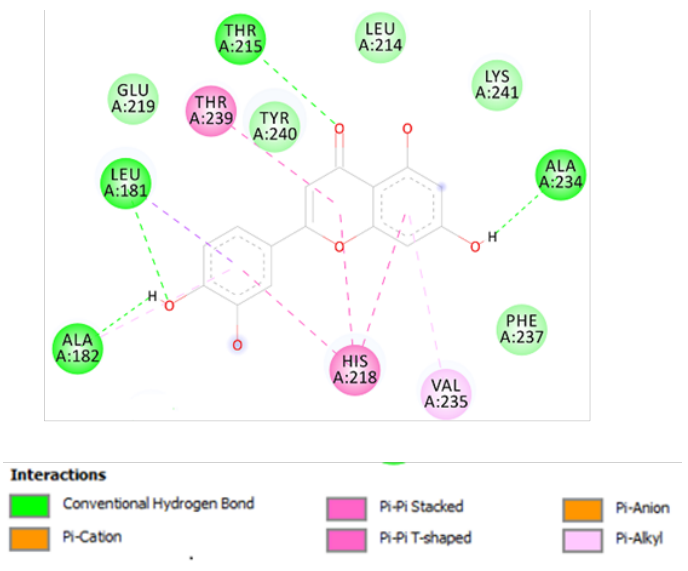

(c)

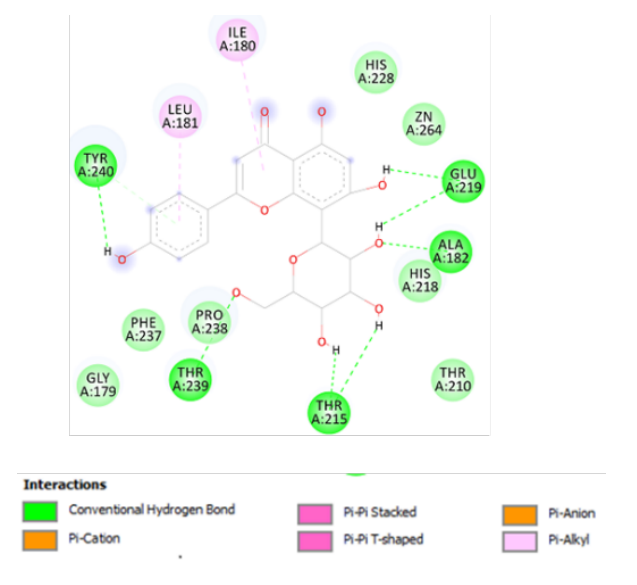

(e)

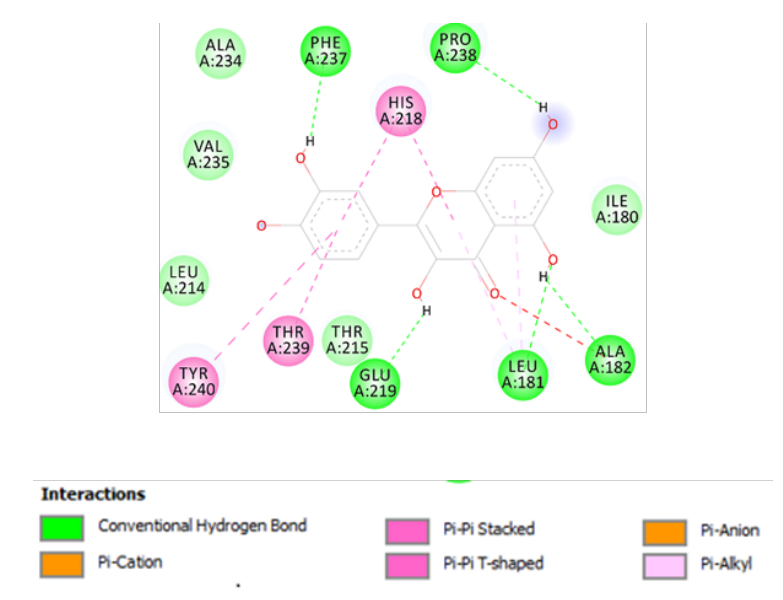

(d)

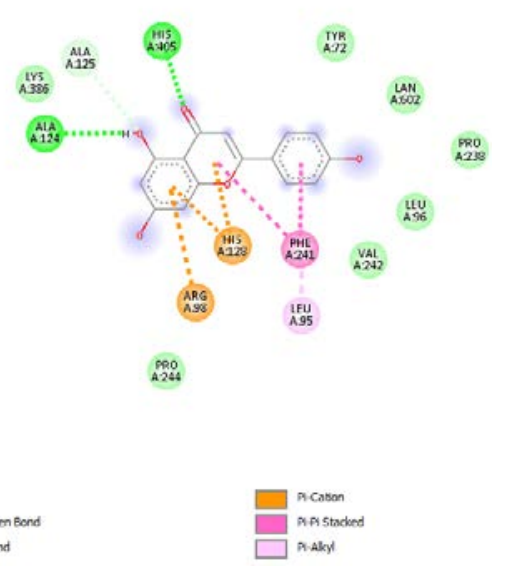

(f)

Figure 8. (a) Putative interactions between apigenin and MMP-12 (PDB: 3F17). Free energy of binding $(\Delta \mathrm{G})$ and affinity $(\mathrm{Ki})$ are $-8.3 \mathrm{kcal} / \mathrm{mol}$ and $0.8 \mu \mathrm{M}$, respectively. (b) Putative interactions between isovitexin and MMP-12 (PDB: 3F17). Free energy of binding $(\Delta \mathrm{G})$ and affinity (Ki) are $-8.8 \mathrm{kcal} / \mathrm{mol}$ and $0.4 \mu \mathrm{M}$, respectively. (c) Putative interactions between luteolin and MMP-12 (PDB: 3F17). Free energy of binding $(\Delta \mathrm{G})$ and affinity $(\mathrm{Ki})$ are $-8.7 \mathrm{kcal} / \mathrm{mol}$ and $0.4 \mu \mathrm{M}$, respectively. (d) Putative interactions between quercetin and MMP-12 (PDB: 3F17). Free energy of binding $(\Delta \mathrm{G})$ and affinity (Ki) are $-8.7 \mathrm{kcal} / \mathrm{mol}$ and $0.4 \mu \mathrm{M}$, respectively. (e) Putative interactions between vitexin and MMP-12 (PDB: 3F17). Free energy of binding $(\Delta \mathrm{G})$ and affinity $(\mathrm{Ki})$ are $-8.8 \mathrm{kcal} / \mathrm{mol}$ and $0.3 \mu \mathrm{M}$, respectively. (f) Putative interactions between apigenin and lanosterol-14-alpha demethylase (PDB: 4LXJ). Free energy of binding $(\Delta \mathrm{G})$ and affinity $(\mathrm{Ki})$ are $-8.8 \mathrm{kcal} / \mathrm{mol}$ and $0.4 \mu \mathrm{M}$, respectively. 

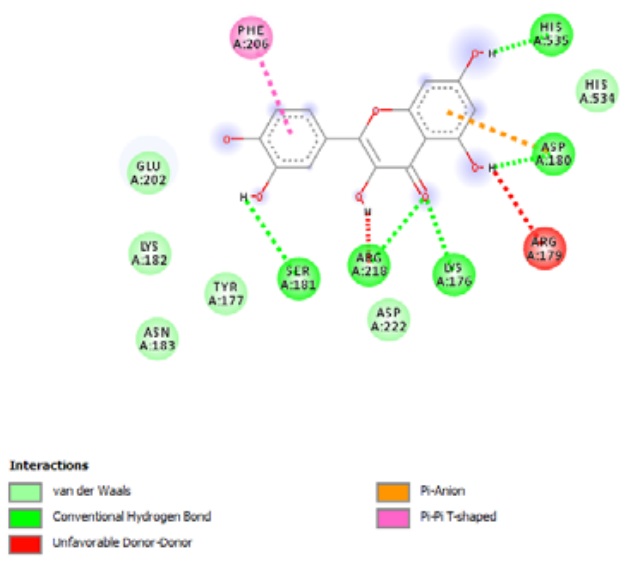

(a)
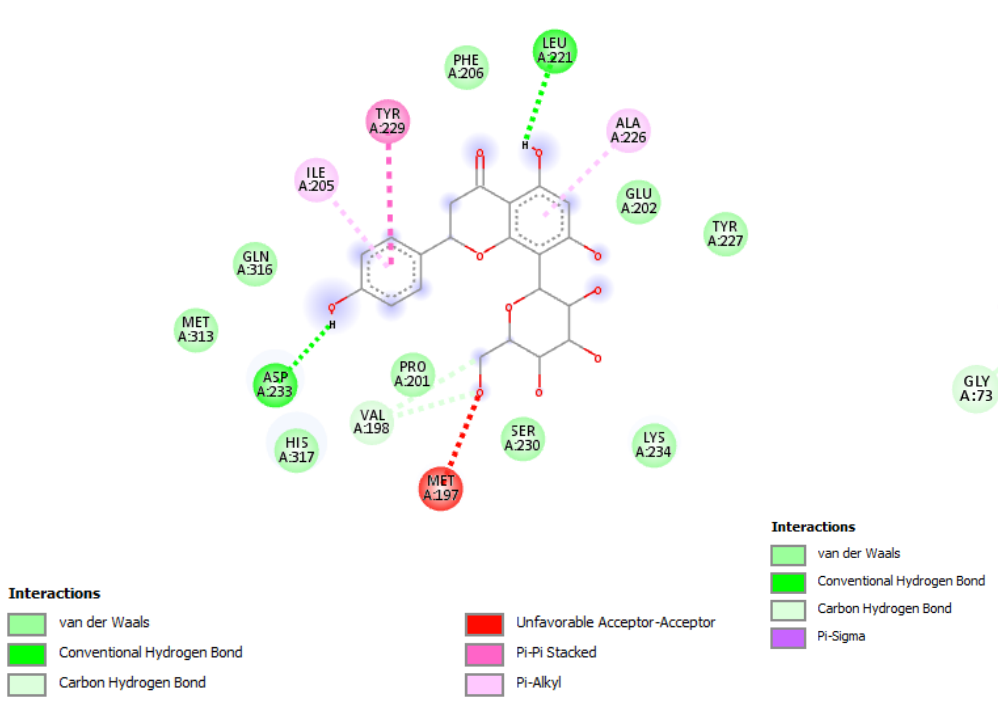

(c)
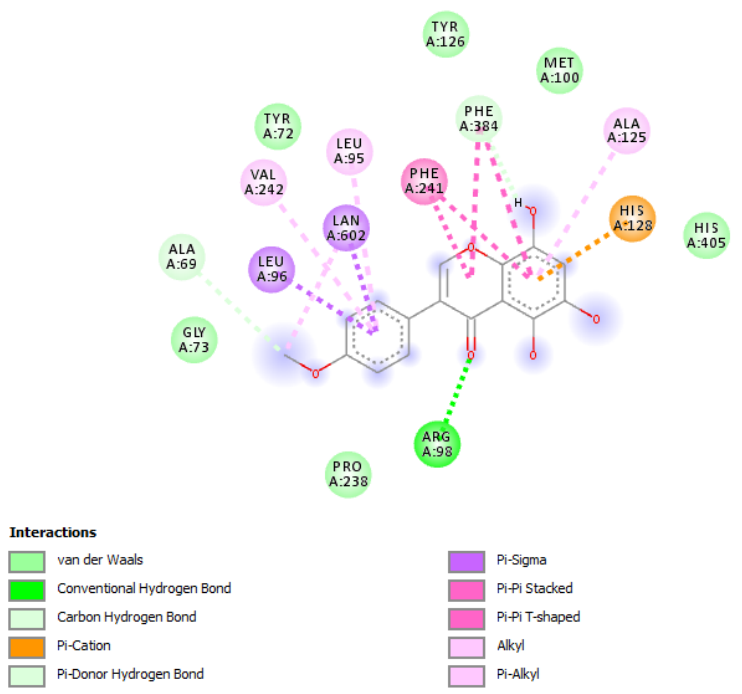

(e)

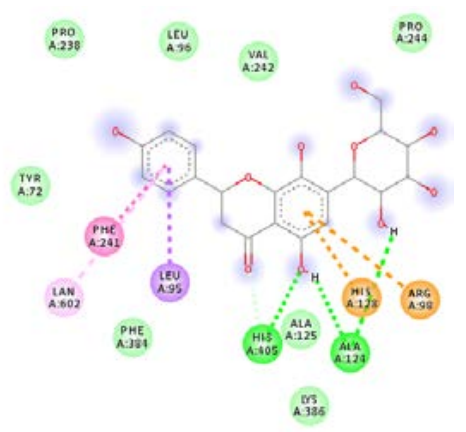

(b)

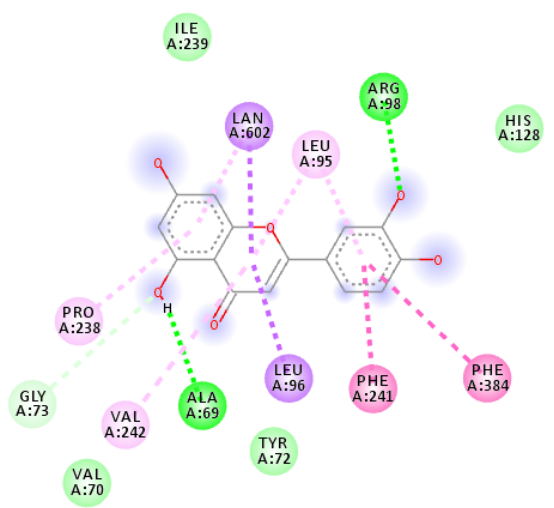

(d)

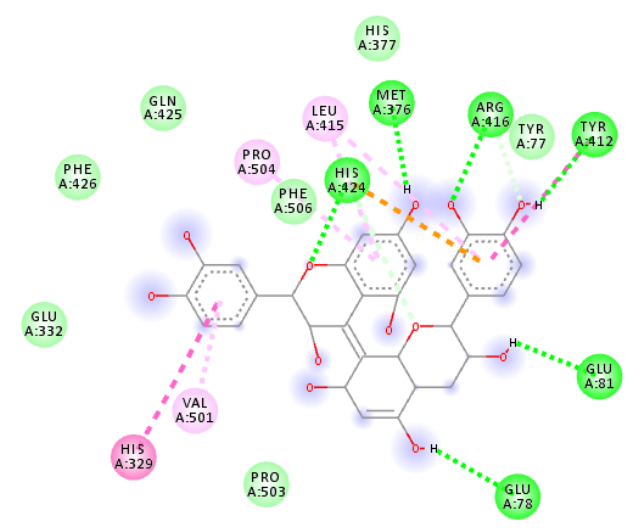

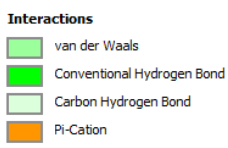

(f)

Figure 9. (a) Putative interactions between quercetin and lanosterol-14-alpha demethylase (PDB: 4LXJ). (b) Putative interactions between isovitexin and lanosterol-14-alpha demethylase (PDB: 4LXJ). Free energy of binding $(\Delta \mathrm{G})$ and affinity (Ki) are $-9.4 \mathrm{kcal} / \mathrm{mol}$ and $0.1 \mu \mathrm{M}$, respectively. (c) Putative interactions 
between vitexin and lanosterol-14-alpha demethylase (PDB: 4LXJ). Free energy of binding $(\Delta \mathrm{G})$ and affinity $(\mathrm{Ki})$ are $-9.4 \mathrm{kcal} / \mathrm{mol}$ and $0.1 \mu \mathrm{M}$, respectively. (d) Putative interactions between luteolin and lanosterol-14-alpha demethylase (PDB: 4LXJ). Free energy of binding $(\Delta \mathrm{G})$ and affinity (Ki) are $-8.2 \mathrm{kcal} / \mathrm{mol}$ and $1 \mu \mathrm{M}$, respectively. (e) Putative interactions between methoxy-trihydroxy(iso)flavone and lanosterol-14-alpha demethylase (PDB: 4LXJ). Free energy of binding $(\Delta \mathrm{G})$ and affinity $(\mathrm{Ki})$ are $-8.7 \mathrm{kcal} / \mathrm{mol}$ and $0.4 \mu \mathrm{M}$, respectively. (f) Putative interactions between procyanidin B and lanosterol-14-alpha demethylase (PDB: 4LXJ). Free energy of binding $(\Delta \mathrm{G})$ and affinity $(\mathrm{Ki})$ are $-8.3 \mathrm{kcal} / \mathrm{mol}$ and $0.8 \mu \mathrm{M}$, respectively.

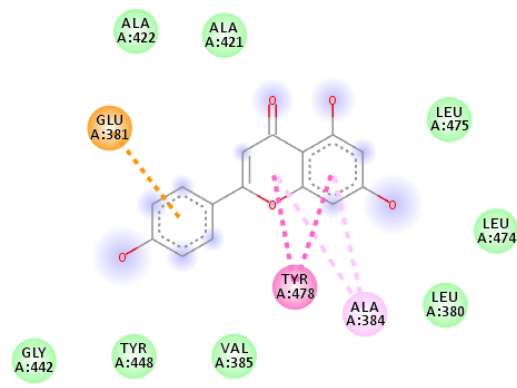
Interactions
$\square$ van der Waal
Pi-Anion

teractions

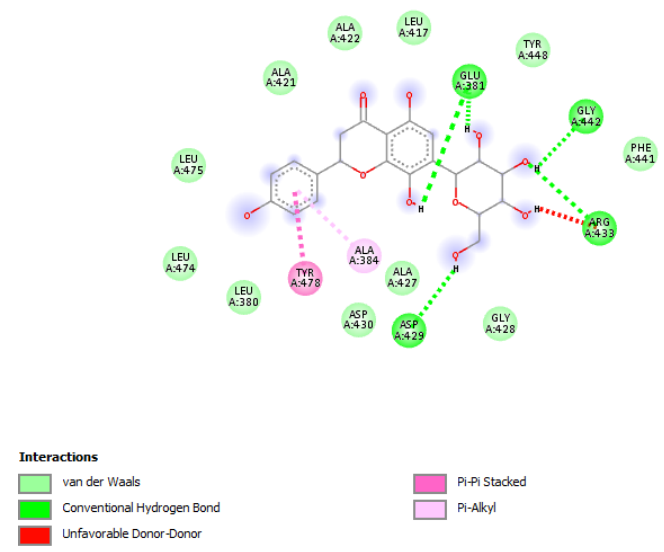

Unfavorable Donor-Donor

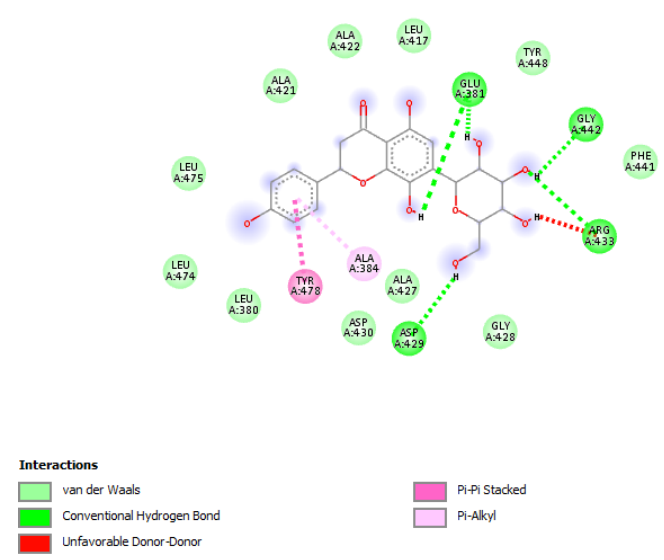

(a)

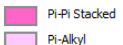

(c)
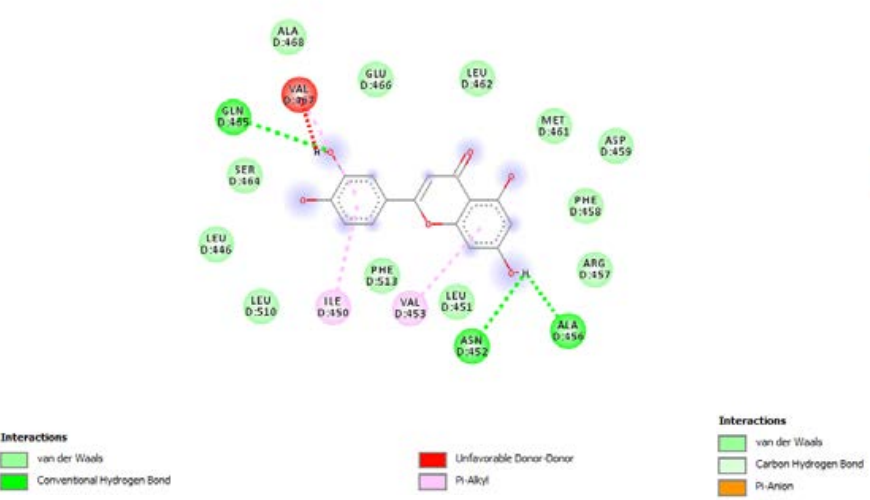

(e)
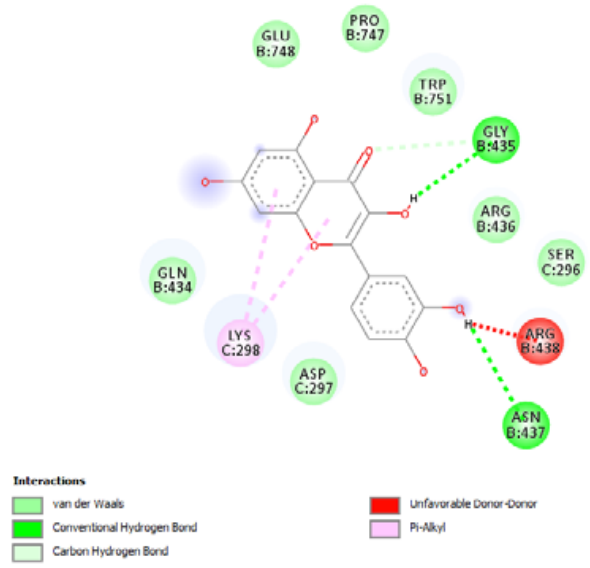

(b)

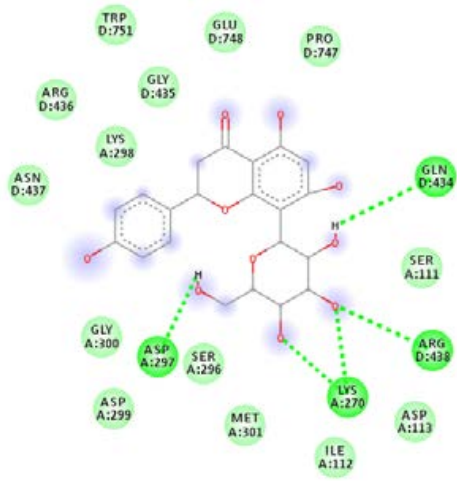

(d)
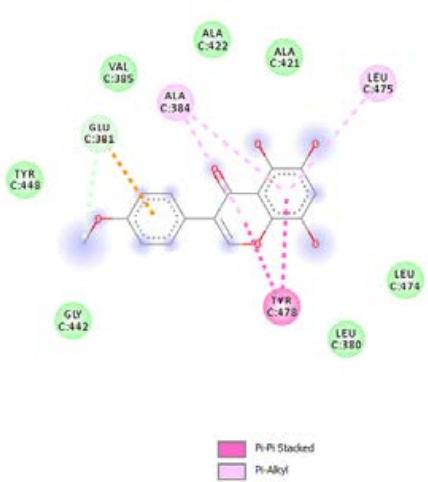

(f)

Figure 10. Cont. 


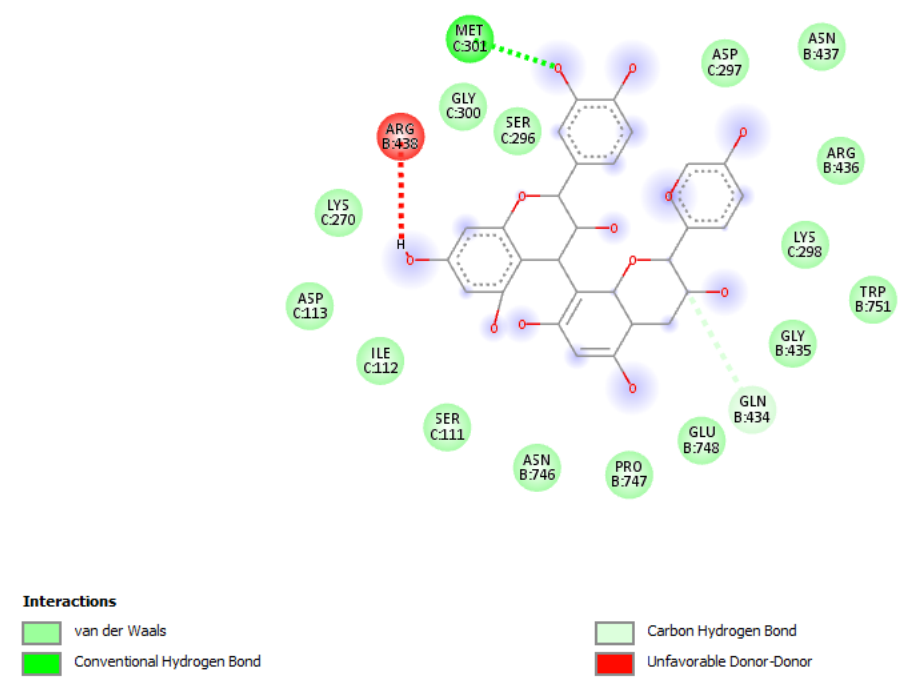

(g)

Figure 10. (a) Putative interactions between apigenin and E. coli gyrase (PDB: 6RKS). Free energy of binding $(\Delta \mathrm{G})$ and affinity $(\mathrm{Ki})$ are $-7.3 \mathrm{kcal} / \mathrm{mol}$ and $4.5 \mu \mathrm{M}$, respectively. (b) Putative interactions between quercetin and $E$. coli gyrase (PDB: 6RKS). Free energy of binding $(\Delta \mathrm{G})$ and affinity (Ki) are $-7.2 \mathrm{kcal} / \mathrm{mol}$ and $5.4 \mu \mathrm{M}$, respectively. (c) Putative interactions between isovitexin and $E$. coli gyrase (PDB: 6RKS). Free energy of binding $(\Delta \mathrm{G})$ and affinity $(\mathrm{Ki})$ are $-8.4 \mathrm{kcal} / \mathrm{mol}$ and $0.7 \mu \mathrm{M}$, respectively. (d) Putative interactions between vitexin and E. coli gyrase (PDB: 6RKS). Free energy of binding $(\Delta G)$ and affinity (Ki) are $-8.5 \mathrm{kcal} / \mathrm{mol}$ and $0.6 \mu \mathrm{M}$, respectively. (e) Putative interactions between luteolin and E. coli gyrase (PDB: 6RKS). Free energy of binding $(\Delta \mathrm{G})$ and affinity $(\mathrm{Ki})$ are $-7.7 \mathrm{kcal} / \mathrm{mol}$ and 2.3 $\mu \mathrm{M}$, respectively. (f) Putative interactions between methoxy-trihydroxy(iso)flavone and $E$. coli gyrase (PDB: 6RKS). Free energy of binding $(\Delta \mathrm{G})$ and affinity $(\mathrm{Ki})$ are $-6.9 \mathrm{kcal} / \mathrm{mol}$ and $8.9 \mu \mathrm{M}$, respectively. (g) Putative interactions between procyanidin B and E. coli gyrase (PDB: 6RKS). Free energy of binding $(\Delta \mathrm{G})$ and affinity $(\mathrm{Ki})$ are $-8.0 \mathrm{kcal} / \mathrm{mol}$ and $1.4 \mu \mathrm{M}$, respectively.

Overall, the results of tyrosinase inhibition assay and bioinformatic analyses suggest the extracts of $R$. racemosa as sources of phytochemicals with promising applications in inflammatory and infectious skin disorders. Among the tested compounds, isovitexin could be the most promising phytochemical, with $\mathrm{Ki}$ values calculated towards the selected enzymes, in the nanomolar range between -9.4 and $-8.4 \mathrm{kcal} / \mathrm{mol}$, corresponding to submicromolar Ki values. This could be related, at least partially, to the higher number of favorable non-covalent bonds predicted by docking analysis.

\section{Discussion}

Data collected in this present study highlighted the key role of extraction techniques and solvent choice in the quest of phytochemicals from $R$. racemosa. Overall it was demonstrated that methanol was a better extraction medium irrespective of the extraction technique used. In terms of antioxidant assays, both methanolic leaf and bark extracts exhibited remarkable reducing potential. Plants are considered as major biological factories for anti-oxidants having the potential to scavenge and detoxify reactive oxygen species (ROS) [37]. ROS are the major precursors of the development of chronic diseases namely diabetes mellitus, cancer, inflammatory disorders, cardiovascular diseases among others [38,39]. On this note, it is acknowledged that anti-oxidants are essential in preventing ROS from initiating diseases. Interestingly, several phytochemicals are reported to possess potent antioxidant capacities due to their ability to donate electrons and/or chelate transition metals [40].

Furthermore, our study also explored the enzymatic inhibitory activities of the extracts. Medicinal plants are abounded with secondary metabolites having broad-spectrum of enzyme inhibitory potentials [41,42]. It is thus of paramount importance to continue searching for inhibitors to tune down activities of enzymes which subsequently prevent initiation and/or progression of diseases. 
Hence, the focus of numerous researchers has now geared towards plants to successfully develop potential enzyme inhibitors. Herein, the enzymatic inhibitory effects of the mangrove plant, R. racemosa were investigated against key enzymes responsible for developing chronic diseases such as diabetes mellitus type II, neurodegenerative disorders and skin hyperpigmentation. As far as our literature search could reach, this report is second-to-none in appraising the enzymatic inhibitory potential of $R$. racemosa. It is of noteworthy interest to highlight that the highest enzyme inhibitory effects were observed against tyrosinase. In this context, bioinformatics analysis predicted putative interactions between tyrosinase and multiple secondary metabolites including apigenin, luteolin, vitexin, isovitexin, procyanidin $B$, quercetin and methoxy-trihydroxy(iso)flavone. The same compounds were also docked against lanosterol-14 $\alpha$-demethylase and E. Coli DNA gyrase, yielding affinities in the submicromolar-micromolar range that further support the observed anti-microbial effects exerted by the extracts. Regarding the antibacterial activity, the selective efficacy against $E$. coli exerted by static maceration extract could support the traditional use of mangrove as a source of products to treat intestinal disorders, such as diarrhea [43]. Similarly, the same extracts, also derived from bark are potential candidates to treat infections caused by $S$. aureus that are frequently related to skin diseases such as abscesses [26]. The extracts were also active against anti-mycotic agents. T. viride, which is well known for its antagonistic ability towards plant pathogenic fungi and received considerable attention as a biocontrol agent of soil-borne plant pathogens [44]. The production of secondary metabolites by Trichoderma strains also shows a great variety and applicative potential in medicine as anti-microbial and anti-fungal agents and as well in agriculture, where the main application is as biocontrol and biofertilizer. In terms of strains susceptibility, the effects on A. ochraceus, one of the main contaminants of foodstuff such as rice, wheat or maize [45], the results were interesting.

\section{Conclusions}

As a conclusion, the leaves and barks of $R$. racemosa represent a potential storehouse of secondary metabolites with promising biological activities for the development of novel pharmacophores. However, further investigation is required to evaluate the toxicity, efficacy and bioavailability of the observed pharmacological effects.

Supplementary Materials: The following are available online at http://www.mdpi.com/2076-3921/9/6/533/s1.

Author Contributions: Conceptualization, C.F., G.Z., G.O., P.A. and L.M.; methodology, C.F., G.Z., G.O., P.A. and L.M.; C.F., G.Z., G.O., P.A. and L.M.; C.F., G.Z., G.O., P.A. and L.M.; C.F., G.Z., G.O., P.A. and L.M.; investigation, R.V., A.C., L.R., S.L., K.I.S., S.C., G.A.F., N.B.S., O.K.E., Z.C., J.J., J.G., M.S. and H.H.A.; resources, C.F., G.Z., G.O., L.M.; C.F., G.Z., G.O., P.A. and L.M.; writing-original draft preparation, M.F.M.; C.F., G.Z., G.O., P.A., A.C. and L.M.; visualization, L.B.; supervision, L.B.; project administration, G.O., C.F. and L.M.; funding acquisition, C.F., L.M., G.O. and A.C. All authors have read and agreed to the published version of the manuscript.

Funding: The study was supported by the Italian Ministry of University funds (FAR 2019) granted to Giustino Orlando, Annalisa Chiavaroli, Luigi Menghini and Claudio Ferrante. This work has been supported by Ministry of Education, Science and Technological Development of Republic of Serbia (451-03-68/2020-14/200007) granted to J.G. and M.S.

Conflicts of Interest: The authors declare no conflict of interest.

\section{References}

1. Gong, B.; Cao, H.; Peng, C.; Perčulija, V.; Tong, G.; Fang, H.; Wei, X.; Ouyang, S. High-throughput sequencing and analysis of microbial communities in the mangrove swamps along the coast of Beibu Gulf in Guangxi, China. Sci. Rep. 2019, 9, 9377. [CrossRef] [PubMed]

2. Bandaranayake, W.M. Bioactivities, bioactive compounds and chemical constituents of mangrove plants. Wetl. Ecol. Manag. 2002, 10, 421-452. [CrossRef]

3. Sur, T.K.; Hazra, A.; Hazra, A.K.; Bhattacharyya, D. Antioxidant and hepatoprotective properties of Indian Sunderban mangrove Bruguiera gymnorrhiza L. leaf. J. Basic Clin. Pharm. 2016, 7, 75-79. [CrossRef] 
4. $\quad$ Bibi, S.N.; Fawzi, M.M.; Gokhan, Z.; Rajesh, J.; Nadeem, N.; Rengasamy, K.R.R.; Albuquerque, R.D.D.G.; Pandian, S.K. Ethnopharmacology, Phytochemistry, and Global Distribution of Mangroves-A Comprehensive Review. Mar. Drugs 2019, 17, 231. [CrossRef] [PubMed]

5. Tomlinson, P.B. Family: Rhizophoraceae. In The Botany of Mangroves, 2nd ed.; Tomlinson, P.B., Ed.; Cambridge University Press: Cambridge, UK, 2016; pp. 315-355.

6. Angalabiri-Owei, B.; Isirima, J. Evaluation of the lethal dose of the methanol extract of Rhizophora racemosa leaf using karbers method. Afr. J. Cell Pathol 2014, 2, 65-68. [CrossRef]

7. Dossou-Yovo, H.; Fifanou, G.; Sinsin, B. Ethnobotanical survey of mangrove plant species used as medicine from ouidah to grand-popo districts, southern Benin. Am. J. Ethnomed 2017, 4, 1-6.

8. Balasubramanian, V.; Rajesh, P.; Rajaram, R.; Kannan, V.R. A review on Rhizophora genus: Therapeutically important perspective phytochemical constituents. Bioactive Phytochemicals: Perspectives for Modern Medicine; Gupta, V.K., Ed.; Daya Publishing House: New Delhi, India, 2015; p. 3.

9. Ukoima, H.; Ikata, M.; Pepple, G. Control of Lasiodiplodia theobromae (PAT) on Rhizophora racemosa using plants extracts. Am. J. Biotechnol. Mol. Sci. 2013, 3, 1-7. [CrossRef]

10. Udeozo, I.; Okafor, G.; Ike, O.; Eze, E. The efficacy of Rhizophora racemosa wood: An important study. Fuw Trend. Sci. Technol. J. 2018, 3, 977-980.

11. List. 2013. Available online: http://www.theplantlist.org/1.1/browse/A/Rhizophoraceae/Rhizophora/ (accessed on 14 April 2020).

12. Chiavaroli, A.; Recinella, L.; Ferrante, C.; Locatelli, M.; Macchione, N.; Zengin, G.; Leporini, L.; Leone, S.; Martinotti, S.; Brunetti, L.; et al. Crocus sativus, Serenoa repens and Pinus massoniana extracts modulate inflammatory response in isolated rat prostate challenged with LPS. J. Biol. Regul. Homeost. Agents 2017, 31, 531-541.

13. Ferrante, C.; Recinella, L.; Ronci, M.; Menghini, L.; Brunetti, L.; Chiavaroli, A.; Leone, S.; Di Iorio, L.; Carradori, S.; Tirillini, B.; et al. Multiple pharmacognostic characterization on hemp commercial cultivars: Focus on inflorescence water extract activity. Food Chem. Toxicol. 2019, 125, 452-461. [CrossRef]

14. Zengin, G.; Locatelli, M.; Stefanucci, A.; Macedonio, G.; Novellino, E.; Mirzaie, S.; Dvorácskó, S.; Carradori, S.; Brunetti, L.; Orlando, G.; et al. Chemical characterization, antioxidant properties, anti-inflammatory activity, and enzyme inhibition of Ipomoea batatas L. leaf extracts. Int. J. Food Prop. 2017, 20, 1907-1919. [CrossRef]

15. Mollica, A.; Zengin, Z.; Stefanucci, A.; Ferrante, C.; Menghini, L.; Orlando, G.; Brunetti, L.; Locatelli, M.; Dimmito, M.P.; Novellino, E.; et al. Nutraceutical potential of Corylus avellana daily supplements for obesity and related dysmetabolism. J. Funct. Foods 2018, 47, 562-574. [CrossRef]

16. Zengin, G.; Aktumsek, A.; Ceylan, R.; Uysal, S.; Mocan, A.; Guler, G.O.; Mahomoodally, M.F.; Glamočlija, J.; Ćirić, A.; Soković, M. Shedding light on the biological and chemical fingerprints of three Achillea species (A. biebersteinii, A. millefolium and A. teretifolia). Food Funct. 2017, 8, 1152-1165. [CrossRef]

17. Garde-Cerdán, T.; Gonzalo-Diago, A.; Pérez-Álvarez, E.P. Phenolic Compounds: Types, Effects and Research; Nova Science Publishers, Incorporated: Hauppauge, NY, USA, 2017.

18. Huang, W.-Y.; Cai, Y.-Z.; Zhang, Y. Natural Phenolic Compounds From Medicinal Herbs and Dietary Plants: Potential Use for Cancer Prevention. Nutr. Cancer 2009, 62, 1-20. [CrossRef] [PubMed]

19. Baba, S.A.; Malik, S.A. Determination of total phenolic and flavonoid content, antimicrobial and antioxidant activity of a root extract of Arisaema jacquemontii Blume. J. Taibah Univ. Sci. 2015, 9, 449-454. [CrossRef]

20. Kumar, S.; Pandey, A.K. Chemistry and biological activities of flavonoids: An overview. Sci. World J. 2013, 2013. [CrossRef] [PubMed]

21. Pereira, G.A.; Molina, G.; Arruda, H.S.; Pastore, G.M. Optimizing the Homogenizer-Assisted Extraction (HAE) of Total Phenolic Compounds from Banana Peel. J. Food Process Eng. 2017, 40, e12438. [CrossRef]

22. Santos, J.S.; Alvarenga Brizola, V.R.; Granato, D. High-throughput assay comparison and standardization for metal chelating capacity screening: A proposal and application. Food Chem. 2017, 214, 515-522. [CrossRef]

23. Zengin, G.; Mahomoodally, M.; Picot-Allain, C.; Cakmak, Y.; Uysal, S.; Aktumsek, A. In vitro tyrosinase inhibitory and antioxidant potential of Consolida orientalis, Onosma isauricum and Spartium junceum from Turkey. S. Afr. J. Bot. 2019, 120, 119-123. [CrossRef]

24. Targher, G.; Lonardo, A.; Byrne, C.D. Nonalcoholic fatty liver disease and chronic vascular complications of diabetes mellitus. Nat. Rev. Endocrinol. 2018, 14, 99. [CrossRef]

25. Zhang, Z.; Zhu, S.; Yang, Y.; Ma, X.; Guo, S. Matrix metalloproteinase-12 expression is increased in cutaneous melanoma and associated with tumor aggressiveness. Tumor Biol. 2015, 36, 8593-8600. [CrossRef] 
26. Becker, R.; Bubeck, J.W. Staphylococcus aureus and the skin: A longstanding and complex interaction. Skinmed 2015, 13, 111-119. [PubMed]

27. Babamahmoodi, F.; Shokohi, T.; Ahangarkani, F.; Nabili, M.; Afzalian Ashkezari, E.; Alinezhad, S. Rare case of Aspergillus ochraceus osteomyelitis of calcaneus bone in a patient with diabetic foot ulcers. Case Rep. Med. 2015, 2015, 1-5. [CrossRef]

28. Bongomin, F.; Batac, C.; Richardson, M.D.; Denning, D.W. A review of onychomycosis due to Aspergillus species. Mycopathologia 2018, 183, 485-493. [CrossRef] [PubMed]

29. Jiang, L.; Li, D.; Shang, P.; Sun, T.; Xiao, X. Preliminary analyses on bacterial diversity and resistance in infection-related skin disorders. Zhonghua Yi Xue Za Zhi 2015, 95, 1762-1764. [PubMed]

30. Lambotte, O.; Fihman, V.; Poyart, C.; Buzyn, A.; Berche, P.; Soumelis, V. Listeria monocytogenes skin infection with cerebritis and haemophagocytosis syndrome in a bone marrow transplant recipient. J. Infect. 2005, 50, 356-358. [CrossRef] [PubMed]

31. Maravić, A.; Rončević, T.; Krce, L.; Ilić, N.; Galić, B.; Čulić Čikeš, V.; Carev, I. Halogenated boroxine dipotassium trioxohydroxytetrafluorotriborate $\mathrm{K} 2[\mathrm{~B} 3 \mathrm{O} 3 \mathrm{~F} 4 \mathrm{OH}]$ inhibits emerging multidrug-resistant and $\beta$-lactamase-producing opportunistic pathogens. Drug Dev. Ind. Pharm. 2019, 45, 1770-1776. [CrossRef]

32. Ranjan, A.; Shaik, S.; Nandanwar, N.; Hussain, A.; Tiwari, S.K.; Semmler, T.; Jadhav, S.; Wieler, L.H.; Alam, M.; Colwell, R.R. Comparative genomics of Escherichia coli isolated from skin and soft tissue and other extraintestinal infections. MBio 2017, 8, e01070-17. [CrossRef] [PubMed]

33. Saikia, L.; Gogoi, N.; Das, P.P.; Sarmah, A.; Punam, K.; Mahanta, B.; Bora, S.; Bora, R. Bacillus cereus-Attributable Primary Cutaneous Anthrax-Like Infection in Newborn Infants, India. Emerging Infect. Dis. 2019, 25, 1261. [CrossRef] [PubMed]

34. Zhang, S.; Liu, X.; Zhang, J.; Cai, L.; Zhou, C. Drug-induced toxic epidermal necrolysis with secondary aspergillus fumigatus infection: A case report. J. Peking Univ. Health Sci. 2019, 51, 977.

35. Houst, J.; Spizek, J.; Havlicek, V. Antifungal Drugs. Metabolites 2020, 10, 106. [CrossRef]

36. Khan, T.; Sankhe, K.; Suvarna, V.; Sherje, A.; Patel, K.; Dravyakar, B. DNA gyrase inhibitors: Progress and synthesis of potent compounds as antibacterial agents. Biomed. Pharmacother. 2018, 103, 923-938. [CrossRef]

37. Smirnoff, N. Antioxidants and Reactive Oxygen Species in Plants; John Wiley \& Sons: Hoboken, NJ, USA, 2008.

38. Davalli, P.; Mitic, T.; Caporali, A.; Lauriola, A.; D'Arca, D. ROS, cell senescence, and novel molecular mechanisms in aging and age-related diseases. Oxid. Med. Cell. Longev. 2016, 2016, 1-18. [CrossRef] [PubMed]

39. Chandrasekaran, A.; Idelchik, M.D.P.S.; Melendez, J.A. Redox control of senescence and age-related disease. Redox Biol. 2017, 11, 91-102. [CrossRef] [PubMed]

40. Segura Campos, M.R.; Ruiz Ruiz, J.; Chel-Guerrero, L.; Betancur Ancona, D. Coccoloba uvifera (L.)(Polygonaceae) fruit: Phytochemical screening and potential antioxidant activity. J. Chem. 2015, 2015, 1-9. [CrossRef]

41. Litalien, C.; Beaulieu, P. Chapter 117-Molecular Mechanisms of Drug Actions: From Receptors to Effectors. In Pediatric Critical Care, 4th ed.; Fuhrman, B.P., Zimmerman, J.J., Eds.; Mosby: Saint Louis, MO, USA, 2011; pp. 1553-1568.

42. Rauf, A.; Jehan, N. Natural products as a potential enzyme inhibitors from medicinal plants. In Enzyme Inhibitors and Activators; InTech: Rijeka, Croatia, 2017; pp. 165-177.

43. Nascimento, D.K.; Souza, I.A.; Oliveira, A.F.D.; Barbosa, M.O.; Santana, M.A.; Pereira, D.F., Jr.; Lira, E.C.; Vieira, J.R. Phytochemical screening and acute toxicity of aqueous extract of leaves of Conocarpus erectus Linnaeus in swiss albino mice. Anais da Academia Brasileira de Ciências 2016, 88, 1431-1437. [CrossRef] [PubMed]

44. Mannina, L.; Segre, A.L.; Ritieni, A.; Fogliano, V.; Vinale, F.; Randazzo, G.; Maddau, L.; Bottalico, A. A new fungal growth inhibitor from Trichoderma viride. Tetrahedron 1997, 53, 3135-3144. [CrossRef]

45. Meng, D.; Garba, B.; Ren, Y.; Yao, M.; Xia, X.; Li, M.; Wang, Y. Antifungal activity of chitosan against Aspergillus ochraceus and its possible mechanisms of action. Int. J. Biol. Macromol. 2020. [CrossRef]

(C) 2020 by the authors. Licensee MDPI, Basel, Switzerland. This article is an open access article distributed under the terms and conditions of the Creative Commons Attribution (CC BY) license (http://creativecommons.org/licenses/by/4.0/). 